Article

\title{
Elastic Stability of Perforated Plates Strengthened with FRP under Uniaxial Compression
}

\author{
Xin Tao, Shuangyin Cao * and Long Zhang
}

Key Laboratory of Concrete and Prestressed Concrete Structures of Ministry of Education, Southeast University, Nanjing 210096, China; 230129148@seu.edu.cn (X.T.); 101000873@seu.edu.cn (L.Z.)

* Correspondence: 101000873@seu.edu.cn; Tel.: +86-158-518-67851

Received: 18 October 2017; Accepted: 13 November 2017; Published: 18 November 2017

\begin{abstract}
Openings are frequently introduced in plates for the purpose of inspection, maintenance, service, etc. The presence of openings reduces the buckling and ultimate capacity significantly, and pasting fiber-reinforced polymers (FRP) is an ideal technique for postponing the buckling and increasing the ultimate capacity of the plates. In this paper, the finite element (FE) method has been employed to study the buckling stress of the perforated plates strengthened with FRP under uniaxial compression, and several parameters are considered: material's geometrical and mechanical properties, boundary conditions, plate aspect ratio, hole sizes, and hole position. Then a method of calculating the buckling stress is proposed and modified based on the theory of composite plate and the numerical results. The study shows that, the stiffness modified factor $\alpha_{D}$, which considers the orthotropic properties of FRP are a function of the reinforcement index $\omega$ and hole size $d / b$ for Boundary conditions (BCs) of $4 \mathrm{~S}$ and 3S1F. And it is recommended to place the big hole close to the middle area of the plate in $x$-axis. It also shows that for a small hole size, there is little effect of the hole position $e_{y} / b$ on buckling coefficient $K_{\mathrm{u}}$ regardless of the $\mathrm{BCs}$, and that effect becomes more pronounced as $d / b$ increases, so it is recommended to put the holes near the middle of the plate for $4 \mathrm{~S}$ and the simple support edge for 3S1F in $y$-axis.
\end{abstract}

Keywords: perforated plates; elastic buckling; fiber-reinforced polymer (FRP); finite element method

\section{Introduction}

Thin steel plate elements are the main structural components of decks and platforms on ships, webs and flanges of plate and box girder bridges, platforms of offshore structures, and architecture structure [1]. Openings are frequently introduced in plates for the purpose of inspection, maintenance, service, etc. [2]. In such cases, the presence of openings redistributes the membrane stresses in the plates and reduces their buckling and compression capacity significantly [3-5]. The reduced buckling strength of the perforated plate may be insufficient to meet the requirements of normal structural serviceability and safety. In this situation, it is necessary and important to develop a method to improve the buckling and ultimate strength of perforated steel plates.

Fiber-reinforced polymer (FRP) has large strength to weight ratios, excellent resistance to corrosion and environmental degradation. It is also very flexible to be formed into any desired shape and easy to be handled during construction. Therefore, FRP has been widely used in strengthening steel structures. In recent years, there have been a number of studies on strengthening steel structures, particularly compressed thin-walled structures. Shaat and Fam [6-9] tested hollow structural steel (HSS) specimens strengthened with carbon fiber-reinforced polymers (CFRP) to study the influence on local and global buckling behavior and column strength. The test indicated that the transverse CFRP layers can help restrain outward directed local buckling of two opposite sides for short columns. Harris [10] employed the strengthening technique of externally bonding FRP sheets to selected sides of steel T-sections in 
order to improve global and local buckling behavior, and the results show that FRP material provides "bracing" against the local buckling. Haedir and Zhao [11] tested ten short cold-formed steel circular hollow section (CHS) columns with externally bonded hoop and longitudinal CFRP, the results show that the reinforcing hoop fibers provide better restraining force to postpone the steel skin buckling when the local slenderness ratio is higher, it also shows that the use of a combination of hoop and longitudinal CFRP in a slender tube can promote the attainment of the yield capacity of the bare tube. Bambach [12-14] tests a CFRP wrapped square hollow sections (SHS) columns with width-to-thickness ratio varying from 12.5 to 120 . The experimental results show that the axial capacity of the wrapped SHS is up to twice of that without wrapping the CFRP, due to the restraining on the development of elastic buckling deflections from the wrapping CFRP. The critical stress is up to four times of that of the steel section due to this restraining effect. Moreover, a design method is developed whereby the theoretical elastic critical stress of the composite steel-CFRP sections developed by Pister and Dong [15] is used to determine the buckling load and the ultimate load.

The previous description shows that openings are frequently introduced in plates and the application of FRP is an ideal technique for postponing the local buckling and increasing the ultimate capacity of the steel structure. However, the researches on pasting FRP sheets for improving the degraded buckling of perforated plates are not investigated in the published literatures. Therefore, the objective of the present paper is to investigate the material's geometrical and mechanical properties, boundary conditions, a plate aspect ratio, hole sizes, and hole position on elastic buckling of perforated plates strengthened with FRP under uniaxial compression. And a calculation method of buckling stress is proposed and modified based on the theory of composite plate and the numerical results.

\section{Problem Definition}

\subsection{Basic Information}

The problem of elastic buckling of perforated plates strengthened with FRP under uniaxial compression (Figure 1) is investigated in this study. The lengths of the plate in the longitudinal and transverse directions are $a$ and $b$ (in this paper, equal to $240 \mathrm{~mm}$ ), respectively. The thicknesses of the steel plate and FRP sheet are $t_{s}$ and $t_{f}$ (single layer of FRP sheet's thickness $t_{f 0}=0.167 \mathrm{~mm}$ and FRP Layers' number is $\left.n_{f}\right)$, respectively. The hole has a diameter $d$, and $e_{x}\left(e_{y}\right)$ is the distance along $x$-axis ( $y$-axis) from the center of the hole to the left (bottom) edge. The elastic modulus of steel and FRP is $E_{f}$ and $E_{s}$, respectively. The Poisson ratio of FRP and steel is $v_{f}$ (equal to 0.25 ) and $v_{s}$ (equal to $0.3)$, respectively.

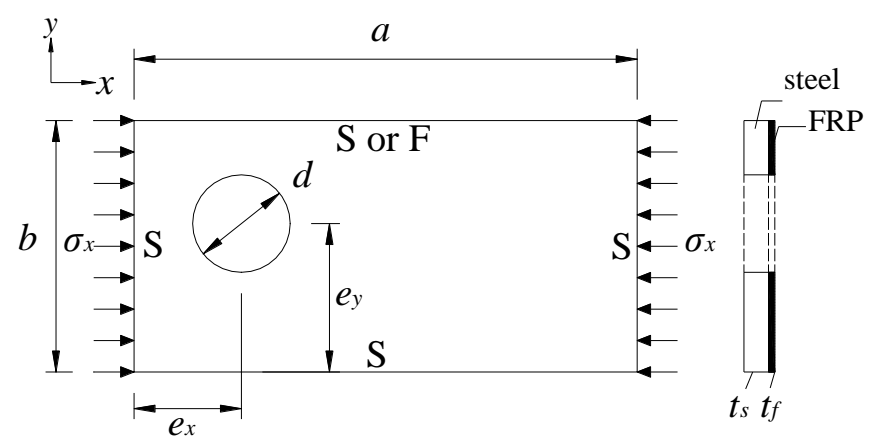

Figure 1. Geometry and Boundary conditions (BCs) of the perforated plates strengthened with fiber-reinforced polymers (FRP).

\subsection{Boundary Conditions (BCs) of the Plate}

The buckling performances of the plate are significantly affected by the BCs. Rectangle, $\mathrm{T}$ and $\mathrm{L}$ sections are the common cross section types used in practical, see in Figure 2. It is noted that mechanical properties of compressed members are derived from the behaviors of the individual plate 
elements that make up the section [14], so the edges between two individual plates are in a state between rotatable and fixed constraint, and the edges are free when the edges have no adjacent edges. The critical case of simply supported edge is selected for conservative calculation for the edges in a state between rotatable and fixed constraint in this paper. Therefore, the columns with rectangle section (Figure 2a) can be seen as four plates with all four edges simple support (4S), the columns with $\mathrm{L}$ and $\mathrm{T}$ sections (Figure $2 \mathrm{~b}, \mathrm{c}$ ) can be seen as two or three plates with three edges simple support and one edge free (3S1F), and both the BCs of $4 \mathrm{~S}$ and $3 \mathrm{~S} 1 \mathrm{~F}$ are considered in this paper, as shown in Figure 1.

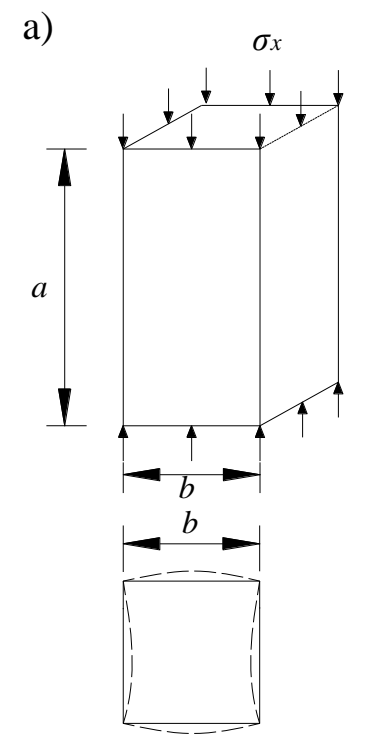

b)

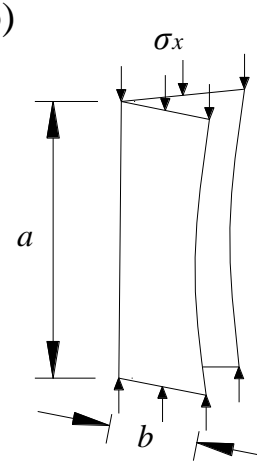

c)
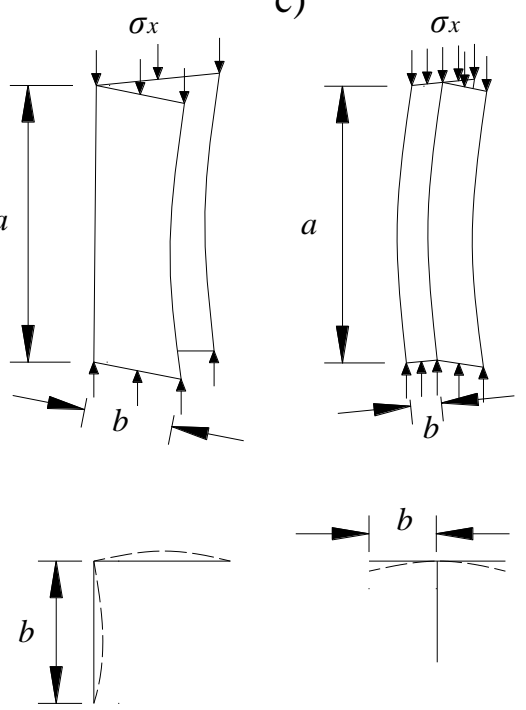

Figure 2. The common cross sections and deformations under compression. (a) Rectangle section; (b) L section; (c) T section.

\subsection{Load Case}

In the present study, only longitudinal uniform uniaxial compression in the $x$ direction is considered, as shown in Figure 1. Other load cases such as biaxial compression and edge shear loading will be discussed in the authors' subsequent research papers. The critical stress $f_{c r}$ is defined by the average stress that is equal to the uniformly applied compressive load divided by the full cross-sectional area assuming no opening within the plate.

\subsection{Parameters Considered in This Study}

The object of the present study is to investigate the buckling of the perforated plates strengthened with FRP under uniaxial compression. The geometric dimensions (such as $t_{f}, t_{s}$ ), and the elastic modulus of FRP $E_{f}$, the BCs of $4 \mathrm{~S}$ and $3 \mathrm{~S} 1 \mathrm{~F}$, the effect of plate aspect ratio $\beta$ (i.e., $a / b$ ), hole size $d / b$, hole position $e_{x} / b$ and $e_{y} / b$ have been considered:

- The parameters of geometrical and mechanical properties such as $t_{f}, t_{s}, E_{f}, E_{s}$ appear in the form $\omega=E_{f} t_{f} / E_{s} t_{s}=E_{f} n_{f} t_{f 0} /\left(E_{s} t_{s}\right)$. In this equation, $E_{f}=1.6 \times 10^{5} \mathrm{MPa}, 2.4 \times 10^{5} \mathrm{MPa}$ and $4.4 \times 10^{5} \mathrm{MPa}, E_{s}=2.0 \times 10^{5} \mathrm{MPa}, n_{f}$ varies between 0 and 3 with an increment of 1 , $t_{f 0}=0.167 \mathrm{~mm}, t_{s}=2 \mathrm{~mm}, 3 \mathrm{~mm}$ and $4 \mathrm{~mm}$.

- The BCs: 4 S and 3S1F.

- $\quad$ Plate aspect ratio $\beta$ : varies between 1 and 4 with increment of 1 .

- Hole size $d / b$ : varies between 0 and 0.7 with an increment of 0.1 , in some case refines to 0.05 .

- Hole position $e_{x} / b$ and $e_{y} / b . e_{x} / b$ varies between 0.05 and $0.5 \beta$ with an increment of $0.0625 . e_{y} / b$ varies between 0.5 and 0.9 with an increment of 0.025 for BCs of $4 \mathrm{~S}$ and between 0.05 and 0.95 with 
an increment of 0.025 for BCs of 3S1F. In all conditions, the distance between hole's edge and plate's edge is no less than $0.05 b$.

\section{The Theory of Composite Plate without Holes}

The buckling problem of rectangular plate subjected to uniaxial compression, was first studied by Timoshenko [16] in 1961 and modified by Gerstle [17] in 1967. Only first order terms in the governing equations were considered, the critical stress $f_{c r}$ at buckling can be written as

$$
f_{c r}=k \frac{\pi^{2}}{t b^{2}} D
$$

where $k$ is a dimensionless buckling coefficient of the plate. $t$ and $b$ are the thickness and width of the plate, respectively. $D$ is the flexural rigidity of the plate, and for the steel plate, it can be written as:

$$
D=\frac{E_{s} t_{s}^{3}}{12\left(1-v_{s}^{2}\right)}
$$

\subsection{The Transformed Flexural Rigidity $D_{t}$}

In 1959, Pister [15] proposed a solution for the transformed flexural rigidity $D_{t}$ of the perfectly bonded layered plate where each layer is assumed to be isotropic, a plate element with the associated coordinate system is shown in Figure 3, and the calculation formulas of $D_{t}$ are in Equations (3)-(6).

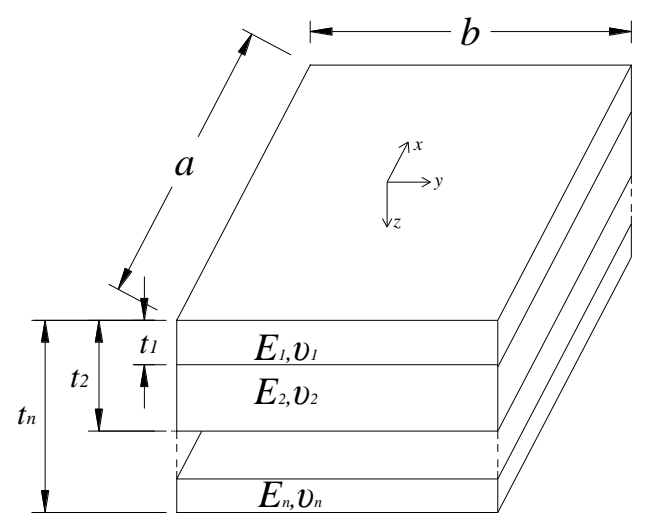

Figure 3. A composite plate with the associated system.

$$
\begin{gathered}
D_{t}=C-\frac{B^{2}}{A} \\
A=\sum_{k} \frac{E_{k}}{1-v_{k}^{2}} t_{k} \\
B=\sum_{k} \frac{E_{k}}{1-v_{k}^{2}} \frac{t_{k}^{2}-t_{k-1}^{2}}{2} \\
C=\sum_{k} \frac{E_{k}}{1-v_{k}^{2}} \frac{t_{k}^{3}-t_{k-1}^{3}}{3}
\end{gathered}
$$

To a pure steel plate, the flexural rigidity of $D_{0}$ can be calculated by Equations (4)-(6), and the rigidity $D_{0}$ can be expressed as Equation (7), which is the same with Equation (2), so the method proposed by Pister can also be used to calculate the flexural rigidity of the pure steel plate.

$$
D_{0}=C_{0}-\frac{B_{0}^{2}}{A_{0}}=\frac{E_{s} t_{s}^{3}}{12\left(1-v_{s}^{2}\right)}
$$


Considering that the steel and CFRP are perfectly bonded up to the ultimate load [18] and the Young's modulus of the adhesive layers is about $1 / 10$ of those of steel and FRP, the effect of adhesive layers is not taken into account for conservative design in this paper. Therefore, a plate strengthened with FRP can be seen as FRP-steel layered plates. Two reinforcement index $\alpha, \gamma$ are introduced as given by Equations (8) and (9).

$$
\begin{gathered}
\alpha=\frac{E_{f}}{1-v_{f}^{2}} / \frac{E_{s}}{1-v_{s}^{2}} \\
\gamma=\frac{t_{f}}{t_{s}}
\end{gathered}
$$

Substitution of Equations (8) and (9) into Equations (4) (6) and then substitution of Equations (4) (6) into (3) leads to Equation (10), where $D_{t}$ is the flexural rigidity of plate strengthened with FRP. Therefore, $D_{t} / D_{0}$ can be expressed as Equation (10), and shown in Figure 4. Equation (10) can be approximate simplified as Equation (11) for calculation convenient, and shown in Figure 5.

$$
\begin{gathered}
D_{t} / D_{0}=1+3 \alpha \gamma\left(1+\frac{4}{3} \gamma^{2}+2 \gamma-\frac{\alpha \gamma(\gamma+1)^{2}}{1+\alpha \gamma}\right) \\
D_{t} / D_{0}=\left\{\begin{array}{c}
1+3 \alpha \gamma, \gamma \leq 0.2 \\
\min \left(1+3 \alpha \gamma, \frac{(\alpha+2)(\gamma+1)^{3}}{3}\right), \text { between } \\
\frac{(\alpha+2)(\gamma+1)^{3}}{3}, 0.3 \leq \gamma \leq 1
\end{array}\right.
\end{gathered}
$$

From the equations, figures and discussion above, the following can be concluded:

- The method proposed by Pister to calculate the transformed flexural rigidity of the perfectly bonded layered plate is available to calculate the flexural rigidity of the pure steel plate.

- When $\gamma$ is in a range of 0 to 0.2 and $\alpha$ is in a range of 0.8 to $2.4, D_{t} / D_{0}$ almost had a linearly related with $\alpha$ and $\gamma$. For $\gamma=0.2, D_{t} / D_{0}$ will increase from 1.48 to 2.44 as $\alpha$ enlarges from 0.8 to 2.4.

- $D_{t} / D_{0}$ will have a non-linear increase as $\gamma$ is enlarged from 0.2 to 0.3 .

- When $\gamma$ is in a range of 0.3 to 1 and $\alpha$ is in a range of 0.8 to 2.4: for a fixed $\alpha, D_{t} / D_{0}$ will had a cubic curvilinear increase as $\gamma$ is enlarged from 0.3 to 1.0 , and for a fixed $\gamma, D_{t} / D_{0}$ will have a linear change as $\alpha$ enlarges from 0.8 to 2.4 . When $\alpha=0.8, D_{t} / D_{0}$ will increase from 2.05 to 7.47 as $\gamma$ enlarges from 0.3 to 1 , when $\alpha=2.4, D_{t} / D_{0}$ will increase from 3.22 to 11.73 as $\gamma$ enlarges from 0.3 to 1 .

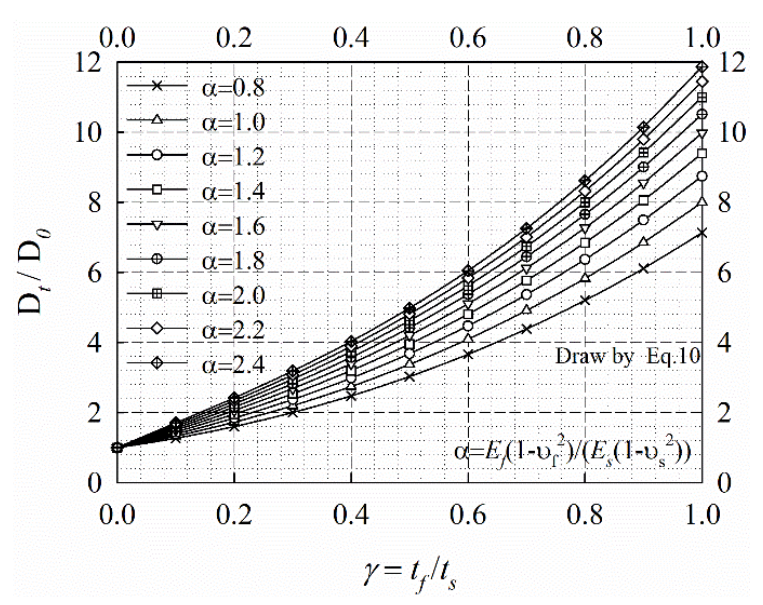

Figure 4. Variation of Stiffness enhancing ratio $D_{t} / D_{0}$ calculated by Equation (10) as a function of $\gamma$. 


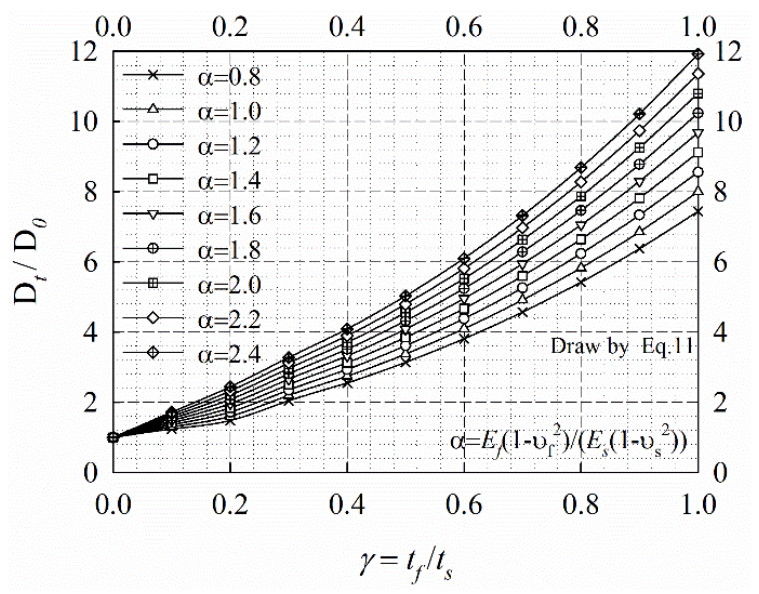

Figure 5. Variation of Stiffness enhancing ratio $D_{t} / D_{0}$ calculated by Equation (11) as a function of $\gamma$.

\subsection{The Buckling Coefficient $k$}

The buckling coefficient $k$ is a dimensionless number factor which depends on the type of loading, $\mathrm{BCs}$, the plate aspect ratio $\beta$ and the openings; and it has nothing to do with whether pasting FRP or not. When the plate aspect ratio is a integer, $k$ is equal to 4 and $0.456+(b / a)^{2}$ for plates with BCs of $4 S$ and 3S1F, respectively [16].

\subsection{Comparison with the Experimental Results}

In order to verify the theoretical method's results, a comparison with the authors' experimental results on the critical stress of perforated plates strengthened with FRP has been performed. As shown in Figure 6, a test rig capable of applying uniaxial compression on the plate was fabricated in the laboratory. And a classic perforated plate strengthened with FRP is shown in Figure 7. The unidirectional FRP has been used. The plates were constructed as following: Firstly, holes are drilled in plates if needed, then a single-layer of FRP is installed directly on the steel surface in the load direction and then another single-layer of FRP is installed in the direction of vertical load. The aim of this paper is to study the buckling of the plate, and when buckling, no delimitation of FRP has been found, so in the finite element (FE) models, the delimitation of FRP and the influence of manufacturing technology is not taken into account, and the FRP are considered an orthotropic material. The values of the basic geometry and material parameters are in Table 1, and the details of the experiments are in literature [19]. The elastic buckling loads $P_{c r E x p}$ are determined from the experiments by two methods, and averaged; These two methods are the load-axial displacement curve and the load-transverse displacement squared method $[14,20]$. The experimental critical stress $f_{c r E x p}$ can be calculated by $P_{c r E x p} /\left(b t_{t}\right)$, where $t_{t}$ represents the total thickness of the plate. The comparison of experimental and theoretical results are in Table 2. From this table, it can be found that experimental and theoretical results are consistent when $d / b$ and $\gamma$ are equal to 0 . It should be noted that the theoretical method cannot consider the influence of hole, which is a very important parameter to the elastic stability of perforated plates strengthened with FRP under uniaxial compression; so in calculating $f_{c r E q .10}$ and $f_{c r E q .11}, k$ is taken as $K_{u}$, which is obtained by the numerical results in the following section. The values calculated by $f_{c r E q .10}$ and $f_{c r E q .11}$ are very closed, it proves the feasibility of Equation (11) in Calculating $D_{t}$. It also can be seen that when $\beta \neq 0$, the theoretical results of $f_{c r E q .10}$ and $f_{c r E q .11}$ are basically larger than experimental results $f_{c r E x p}$. The reason is that the theory method cannot consider the orthotropic properties of FRP, the effect of FRP's orthotropic properties is more obvious in the following numerical analysis, especially when pasting a lot of FRP. 


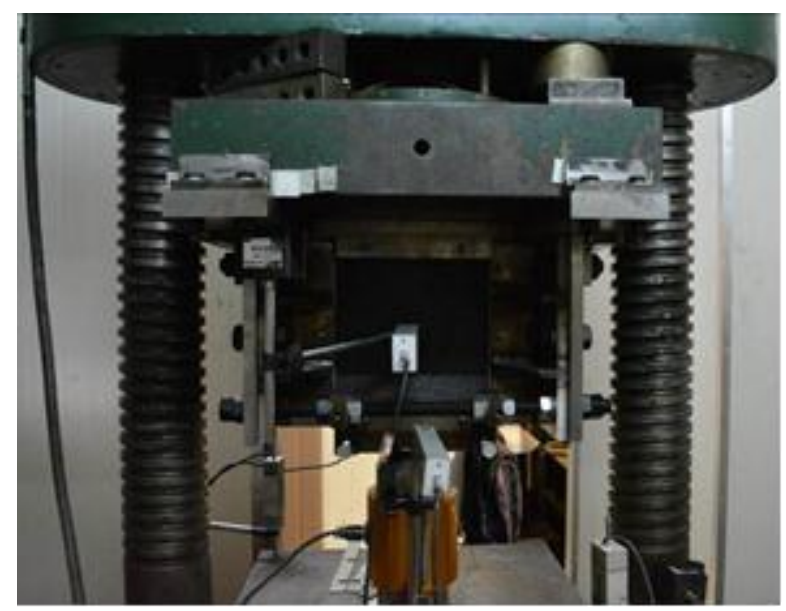

Figure 6. Test rig setup.

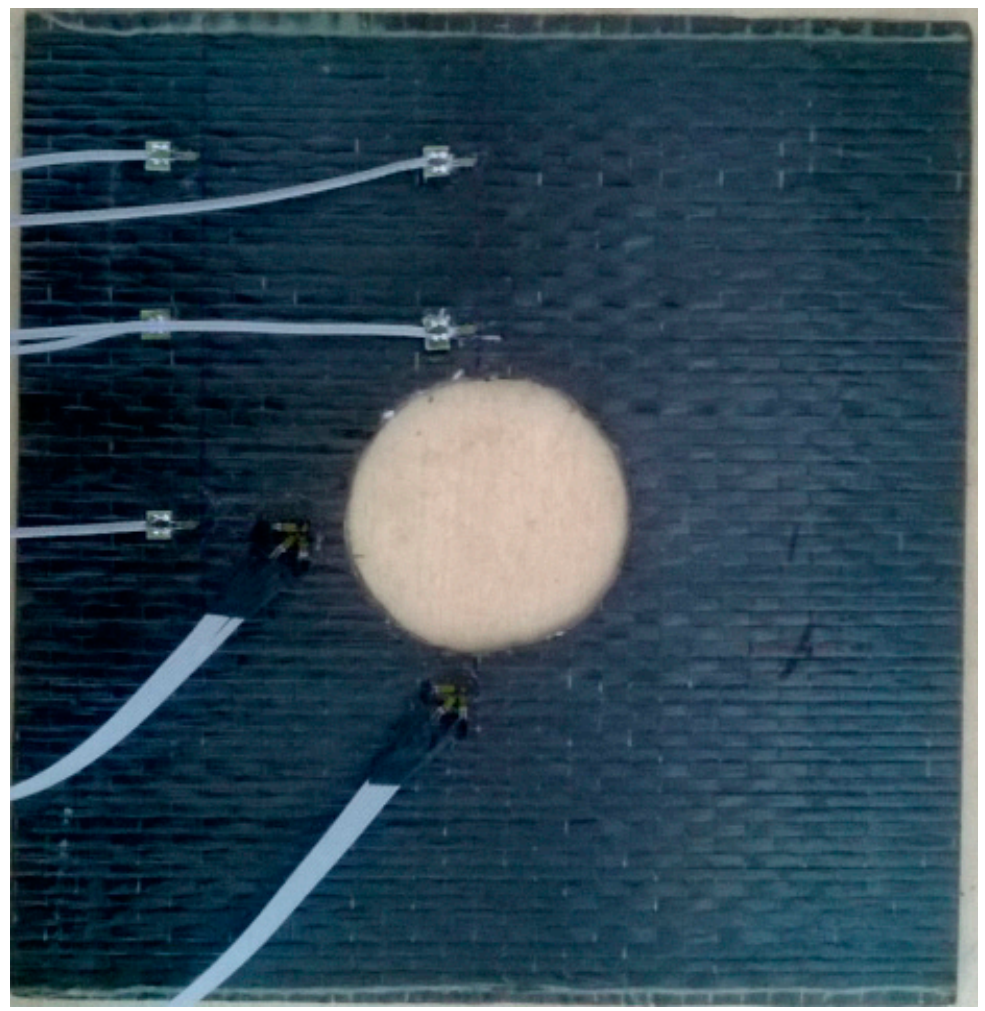

Figure 7. A classic perforated plate strengthened with FRP.

Table 1. The basic geometry and material parameters. BCs: Boundary conditions.

\begin{tabular}{cccccccccc}
\hline $\boldsymbol{a}(\mathbf{m m})$ & $\boldsymbol{b}(\mathbf{m m})$ & $\boldsymbol{t}_{\boldsymbol{s}}(\mathbf{m m})$ & $\boldsymbol{t}_{f 0}(\mathbf{m m})$ & $\left.E_{\boldsymbol{s}} \mathbf{( M P a}\right)$ & $E_{f}(\mathbf{M P a})$ & $v_{s}$ & $v_{f}$ & $f_{y}(\mathbf{M P a})$ & $\mathbf{B C s}$ \\
\hline 240 & 240 & 2.61 & 0.167 & $2.0 \times 10^{5}$ & $2.6 \times 10^{5}$ & 0.3 & 0.25 & 287.9 & $4 S$ \\
\hline
\end{tabular}


Table 2. The comparison of experimental, theoretical, finite element (FE) models and Calculation method in this paper.

\begin{tabular}{|c|c|c|c|c|c|c|c|c|c|c|c|c|c|c|c|c|}
\hline NO. & $d / b$ & $\alpha$ & $\gamma\left(\times 10^{-2}\right)$ & $D_{0}\left(\times 10^{5}\right)$ & $D_{t E q .10}\left(\times 10^{5}\right)$ & $D_{t E q .11}\left(\times 10^{5}\right)$ & $\mathbf{P}_{\text {crExp }}$ & $f_{\text {crExp. }}$ & $f_{\text {crEq. } .17}$ & $f_{c r E q .23}$ & $f_{\text {crFEM }}$ & $\alpha_{D}$ & $K_{u}$ & $f_{\text {crCal }}$ & $\mathbf{P}_{u E x p}$ & $\mathbf{P}_{u C a l}$ \\
\hline 1 & 0 & 1.225 & 0 & 3.35 & 3.35 & 3.35 & 55.83 & 89.13 & 88.08 & 88.08 & 87.79 & 1.000 & 4.00 & 88.08 & 94.02 & 87.59 \\
\hline 2 & 0.1 & 1.225 & 0 & 3.35 & 3.35 & 3.35 & 53.78 & 85.85 & 83.08 * & 83.08 * & 83.06 & 1.000 & 3.77 & 83.08 & 90.11 & 85.41 \\
\hline 3 & 0.3 & 1.225 & 0 & 3.35 & 3.35 & 3.35 & 47.89 & 76.45 & 72.95 * & 72.95 * & 72.93 & 1.000 & 3.31 & 72.95 & 79.66 & 80.71 \\
\hline 4 & 0.5 & 1.225 & 0 & 3.35 & 3.35 & 3.35 & 45.12 & 72.03 & $71.62 *$ & $71.62 *$ & 71.60 & 1.000 & 3.25 & 71.62 & 74.44 & 80.06 \\
\hline 5 & 0 & 1.225 & 6.4 & 3.35 & 4.18 & 4.14 & 64.88 & 97.34 & 103.24 & 102.25 & 95.66 & 0.946 & 4.00 & 96.76 & 101.86 & 97.03 \\
\hline 6 & 0.1 & 1.225 & 6.4 & 3.35 & 4.18 & 4.14 & 61.90 & 92.87 & $97.39 *$ & $96.45 *$ & 89.84 & 0.934 & 3.77 & 90.13 & 96.64 & 94.13 \\
\hline 7 & 0.3 & 1.225 & 6.4 & 3.35 & 4.18 & 4.14 & 58.28 & 87.45 & $85.51 *$ & 84.68 * & 76.08 & 0.910 & 3.31 & 77.13 & 92.72 & 87.99 \\
\hline 8 & 0.5 & 1.225 & 6.4 & 3.35 & 4.18 & 4.14 & 50.54 & 75.83 & 83.95 * & 83.15 * & 72.44 & 0.887 & 3.25 & 73.77 & 78.35 & 86.30 \\
\hline
\end{tabular}

${ }^{*} k$ is taken as $K_{u}$, which is obtained by the numerical results in the following section. 


\subsection{The Proposed of Formula Correction}

From the above comparison and analysis, we know that the stiffness obtained by theoretical calculation is too large due to not consider the orthotropic properties of FRP , so a modified factor of stiffness $\alpha_{D}$ is proposed, we consider $\alpha_{D}$ is a function of $\omega, \beta$ and $d / b$. It also can be seen from above analysis that the theory method cannot consider the influence of hole on buckling, so the buckling coefficient $k$ needs to be modified as $K_{u}$ which considers the change of $d / b, \beta$ and the hole position $e_{x}$ (and $e_{y}$ ). Therefore, the critical stress of perforated plates strengthened with FRP at buckling can be written as Equation (12). The values and change rules of $\alpha_{D}$ and $K_{u}$ are the focuses of the following numerical simulation studies.

$$
f_{c r}=K_{u} \frac{\pi^{2}}{t_{t} b^{2}}\left(\alpha_{D} D_{t}\right)=\alpha_{D} K_{u} \frac{\pi^{2}}{t_{t} b^{2}} D_{t}
$$

\section{Finite Element Model and Verification}

\subsection{Method of Analysis}

The buckling problem of the plate is to solve the eigenvalue, the lowest eigenvalue corresponds to the critical stress while the eigenvector defines the deformed shape at buckling. A general-purpose finite element program, ABAQUS (Version: 6.11, Dassault Systemes S.A, Concord, MA, USA, 2011), has been utilized in the investigation to determine the elastic buckling loads of the perforated plates strengthened with FRP under uniaxial compression.

After performing the eigenvalue analysis, the lowest eigenvalue, $\lambda_{1}$, can be expressed as Equation (13), so $\left(\alpha_{D} K_{u}\right)$ can be expressed as Equation (14). It should be noted that $\alpha_{D}$ is equal to 1 as $\omega=0$ regardless of other parameters, so we let $\omega=0$ to study the variation of $K_{u}$ as $d / b, \beta, e_{x} / b$, $e_{y} / b$ and BCs change. We can change the form of $\alpha_{D}$ into Equation (15) to study the variation of $\alpha_{D}$, where both the molecule and the denominator can be calculated by Equation (14) using numerical simulation results.

$$
\begin{gathered}
\lambda_{1}=\alpha_{D} K_{u} \frac{\pi^{2}}{t_{t} b^{2}} D_{t} \\
\alpha_{D}(\omega, \beta, d / b, B C s) K_{u}\left(d / b, \beta, e_{x} / b, e_{y} / b, B C s\right)=\lambda_{1} \frac{t_{t} b^{2}}{\pi^{2} D_{t}} \\
\alpha_{D}(\omega, \beta, d / b, B C s)=\frac{\alpha_{D}(\omega, \beta, d / b, B C s) K_{u}\left(d / b, \beta, e_{x} / b, e_{y} / b, B C s\right)}{\alpha_{D}(0, \beta, d / b, B C s) K_{u}\left(d / b, \beta, e_{x} / b, e_{y} / b, B C s\right)}
\end{gathered}
$$

\subsection{Model Description}

Four typical perforated plate models with $\beta=1,2,3$ and 4 are shown in Figure 8. The plates have dimensions $a$ and $b$, and circular hole with diameter $d$. The plate was discretized into a mesh consisting of S4R element, each node of the S4R element has six active degrees of freedom (DOF), including three translational DOFs and three rotational DOFs. The mesh size are dependent on the hole sizes and the extensive convergence studies in the following section.

The classical elastic-plastic material and lamina material are used to model the steel and FRP, respectively. The steel-FRP composite section can be easily created by increasing the number of layers and changing the material properties of each layer.

The BCs of the plates considered in this paper are $4 \mathrm{~S}$ and 3S1F. For simple support edge, there will be no lateral edge displacements perpendicular to the plate's plane but rotations about the axis of each edge are allowed. And a uniform load of magnitude $t_{t}$ per unit width is applied perpendicular to the width edges. 


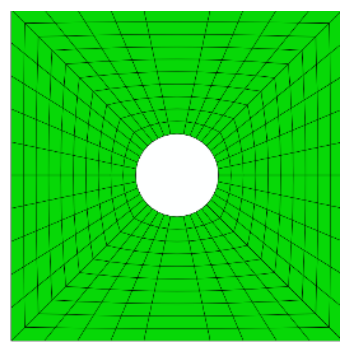

(a)

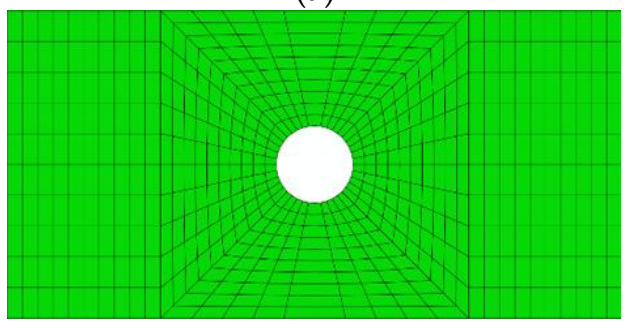

(b)

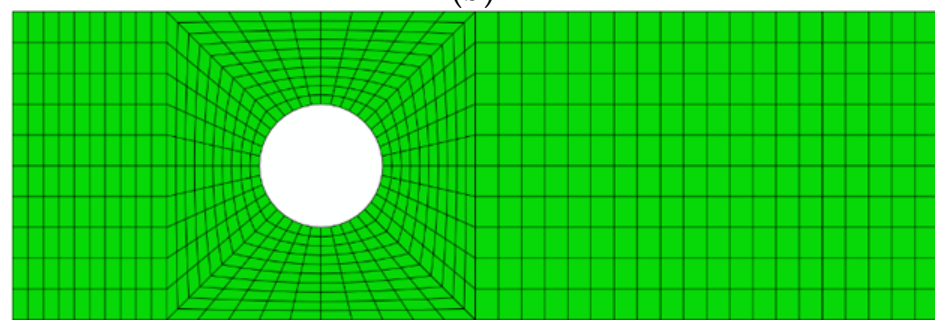

(c)

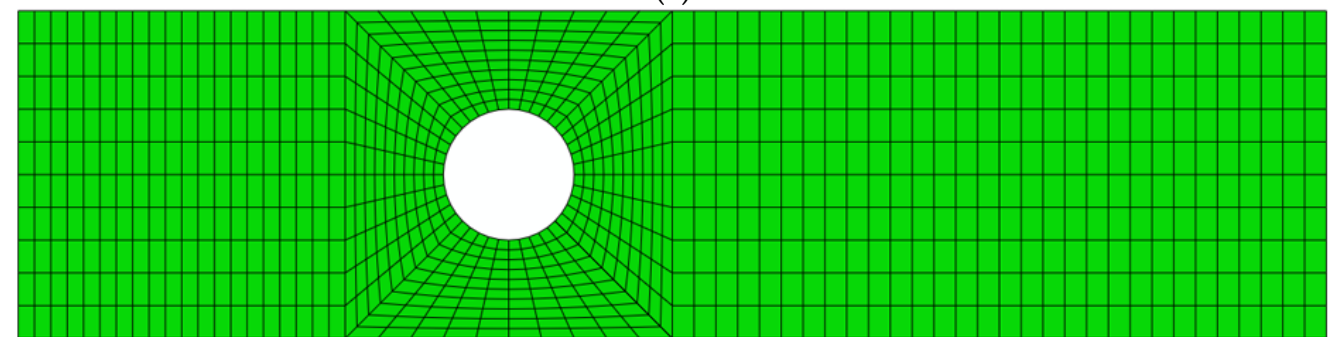

(d)

Figure 8. Examples of four typical plate models' meshes. (a) case of $\beta=1$; (b) case of $\beta=2$; (c) case of $\beta=3 ;(\mathbf{d})$ case of $\beta=4$.

\subsection{Model Verification}

A mesh study is conducted to observe the convergence of buckling stress as the mesh size increases. Take plate with No. 8 in Table 2 for example, the Eigenvalues with respect to element numbers depended on mesh size, as shown in Figure 9. It can be seen that the curves starts to converge after an element number of 400 , which with the general mesh sizes of $b / 10$ and with mesh sizes in hole edge of $\pi d / 40$. In order to reduce computational time, those meshes are adopted in the subsequent buckling analysis.

In order to verify FE models used in this study, a comparison with the authors' experimental results [19] on the critical stress of perforated plates strengthened with FRP has been performed, and the results are in Table 2. It can be seen that the FE models have a very limited relative absolute error in $f_{c r}$, in the consider cases, the errors are $13.0 \%$ on maximum, $4.05 \%$ on average, and 0.108 on the coefficient of variation. Therefore, the FE models are very accurate and enough for the studies in the next section. 


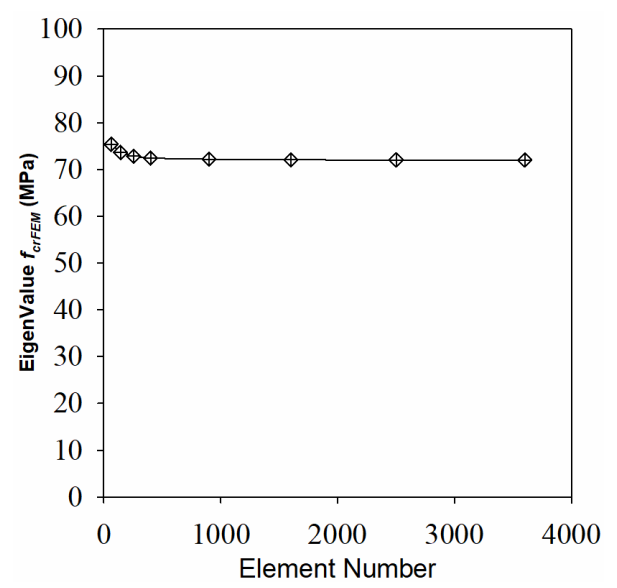

Figure 9. Finite element (FE) models converge using mesh refinement.

\section{Discussion of the Results}

\subsection{Buckling Mode Shapes}

Figures 10-13 show the buckling mode shapes of perforated plates strengthened with FRP under uniaxial compression with different $e_{x} / b, \beta, d / b$ and BCs. The following are found:

When the hole center is in the middle of the plate, the buckling mode shapes transform from two half-waves to three half-waves as $d / b$ is enlarged from 0 to 0.7 (seen from Figure 10), a similar phenomenon occurs in all plates with $e_{x} / b=1$ and 2 .

- From Figures 10a and $11 \mathrm{a}$, it can be seen that when $d / b=0.1$, the buckling mode shapes are very close to plates without hole; and as $d / b$ is enlarged, the max deformations of the plate are firstly centered near the hole then gradually transferred between hole and loading edge for $4 S$ (seen from Figures $10 \mathrm{~b}-\mathrm{d}$ and $11 \mathrm{~b}-\mathrm{d}$ ).

- $\quad$ From Figure 12, it can be seen that as $\beta$ increases, the deformations of the existing part have been basically unchanged, and the deformations of the adding part are very small.

- From Figure 13, it can be seen that the mode shapes are essentially unchanged as $d / b$ is enlarged for plates with BCs of 3S1F.

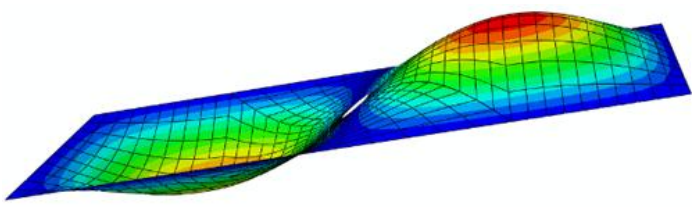

(a)

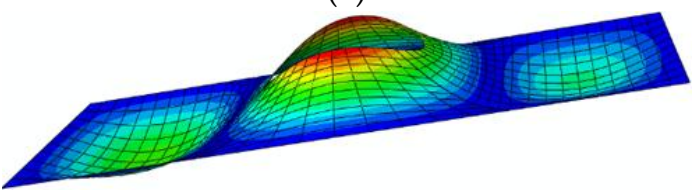

(c)

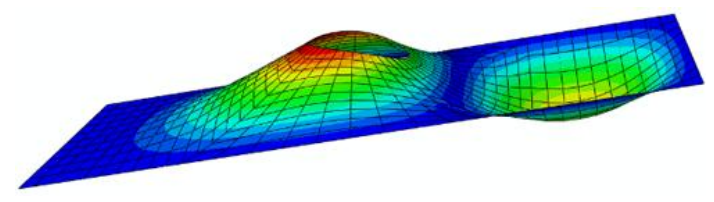

(b)

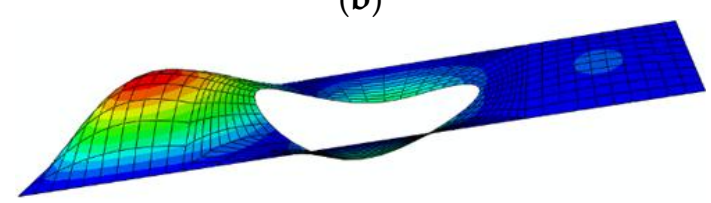

(d)

Figure 10. The model shapes of plates with $e_{x} / b=1, \beta=2$ and BCs of $4 S$. (a) $d / b=0.1 ;(\mathbf{b}) d / b=0.3$; (c) $d / b=0.5 ;$ (d) $d / b=0.7$. 


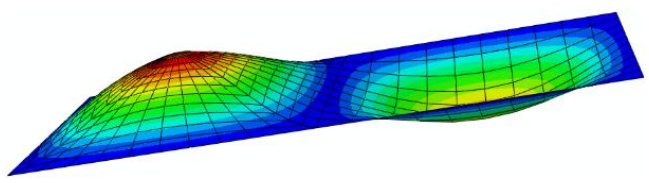

(a)

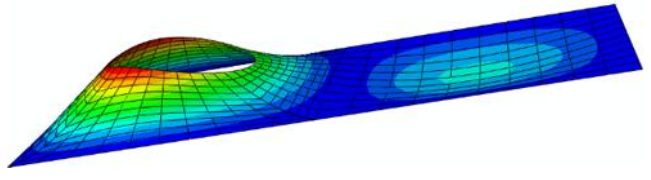

(c)

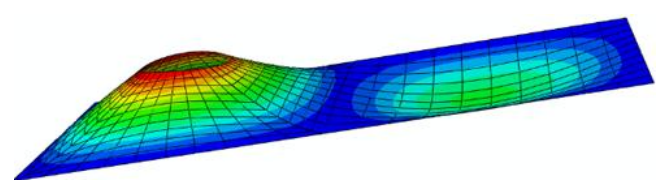

(b)

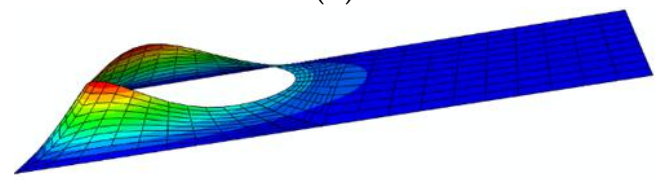

(d)

Figure 11. The model shapes of plates with $e_{x} / b=0.5, \beta=2$ and BCs of $4 S$. (a) $d / b=0.1 ;(\mathbf{b}) d / b=0.3$; (c) $d / b=0.5 ;(\mathbf{d}) d / b=0.7$.

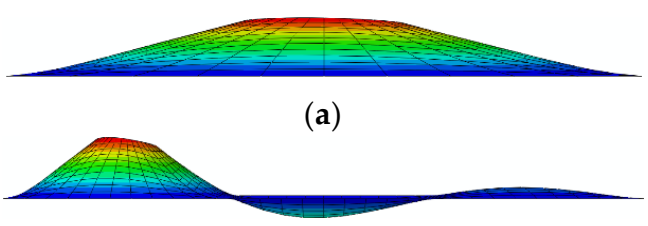

(c)

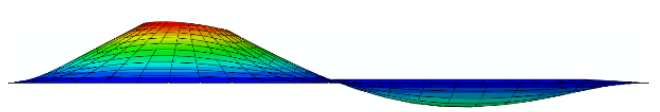

(b)

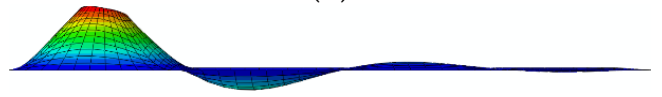

(d)

Figure 12. The model shapes of plates with $e_{x} / b=0.5$, BCs of $4 S$ and different $\beta$ from 1 to 4 . (a) $\beta=1$; (b) $\beta=2$; (c) $\beta=3$; (d) $\beta=4$.

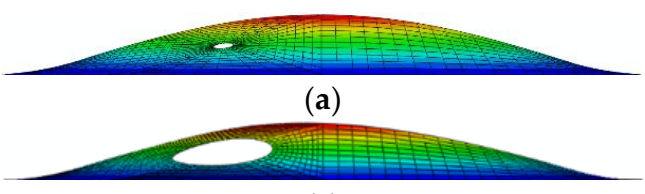

(c)

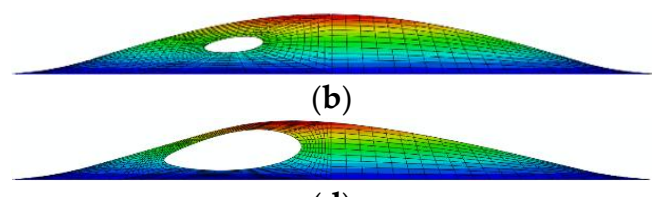

(d)

Figure 13. The model shapes of plates with $e_{x} / b=1, \beta=3$ and BCs of $3 S$. (a) $d / b=0.1 ;(\mathbf{b}) d / b=0.3$; (c) $d / b=0.5 ;$ (d) $d / b=0.7$.

\subsection{The Stiffness Modified Factor $\alpha_{D}$}

Due to the orthotropic properties of FRP, a modified factor of stiffness $\alpha_{D}$ has been proposed. And a reinforcement index of FRP has been defined as:

$$
\omega=\frac{E_{f} t_{f}}{E_{s} t_{s}}=\frac{n_{f} E_{f} t_{f 0}}{E_{s} t_{s}}
$$

In order to study the variation of $\alpha_{D}$, a large number of parametric analysis have been performed in this section, the parameters include reinforcement index $\omega$, plate aspect ratio $\beta$, hole size $d / b$, and BCs. The parametric analysis' results are handled by the above approach in Equations (14) and (15), and plotted in Figures 14 and 15 for BCs of $4 S$ and 3S1F, respectively.

Figure 14 shows the variation of $\alpha_{D}$ as the reinforcement index $\omega$ increases from 0 to 0.8 for different $d / b$ from 0 to 0.7 and different plate aspect ratios $\beta$ from 1 to 4 with the BCs of $4 \mathrm{~S}$. It can be seen that the curves in Figure 14 with the same $d / b$ and different $\beta$ are almost the same and the values in Figure 14a are slightly smaller. Therefore, $\alpha_{D}$ of a perforated rectangular plate strengthened by FRP with an integer aspect ratio could be conservatively taken as that of a perforated square plate, so only plates with $\beta=1$ are analyzed in the following. From Figure $14 \mathrm{a}$, it can be seen that $\alpha_{D}$ decreases as $\omega$ is enlarged and the curves can be coupled by Equation (17), where $m, n$ are the undetermined second-order and first-order coefficients. For different $d / b, \mathrm{~m}$ and $\mathrm{n}$ are calculated in Table 3 , and $R^{2}$ is the correlation coefficient.

$$
\alpha_{D}=m \omega^{2}+n \omega+1
$$


Table 3. The relationship $d / b$ with $\mathrm{m}$ and $\mathrm{n}$.

\begin{tabular}{ccccccccc}
\hline $\boldsymbol{d} / \boldsymbol{b}$ & $\mathbf{0}$ & $\mathbf{0 . 1}$ & $\mathbf{0 . 2}$ & $\mathbf{0 . 3}$ & $\mathbf{0 . 4}$ & $\mathbf{0 . 5}$ & $\mathbf{0 . 6}$ & $\mathbf{0 . 7}$ \\
\hline$m$ & 0.69 & 0.76 & 0.92 & 1.09 & 1.23 & 1.34 & 1.40 & 1.47 \\
$n$ & -0.74 & -0.83 & -1.04 & -1.25 & -1.44 & -1.59 & -1.69 & -1.79 \\
$R^{2}$ & 0.91 & 0.92 & 0.93 & 0.94 & 0.94 & 0.95 & 0.95 & 0.95 \\
\hline
\end{tabular}

In Table 3, $m$ and $n$ can be approximate expressed as Equations (18) and (19), so $\alpha_{D}$ with BCs of $4 S$ can be calculated by Equation (20)

$$
\begin{gathered}
m=1.2(d / b)+0.7 \\
n=-1.6(d / b)-0.74 \\
\alpha_{D}=(1.2 d / b+0.7) \omega^{2}-(1.6 d / b+0.74) \omega+1
\end{gathered}
$$

For the plates with BCs of $3 S$ in Figure 15, the same method can be used to study the variation in $\alpha_{D}$ as the reinforcement index $\omega$ increases from 0 to 0.8 for different plate aspect ratios $\beta$ from 1 to 4 . It also can be seen that $\alpha_{D}$ decreases as $\omega$ is enlarged. It is noted that $\alpha_{D}$ is independent of $d / b$ when $\beta=2,3$ and 4 , so $\alpha_{D}$ with BCs of $3 \mathrm{~S} 1 \mathrm{~F}$ can be calculated by Equation (21).

$$
\alpha_{\mathrm{D}}=\left\{\begin{array}{r}
(0.28 d / b+0.56) \omega^{2}-(0.33 d / b+0.69) \omega+1, \beta=1 \\
1.18 \omega^{2}-1.45 \omega+1, \beta=2 \\
1.42 \omega^{2}-1.73 \omega+1, \beta=3 \\
1.51 \omega^{2}-1.84 \omega+1, \quad \beta=4
\end{array}\right.
$$
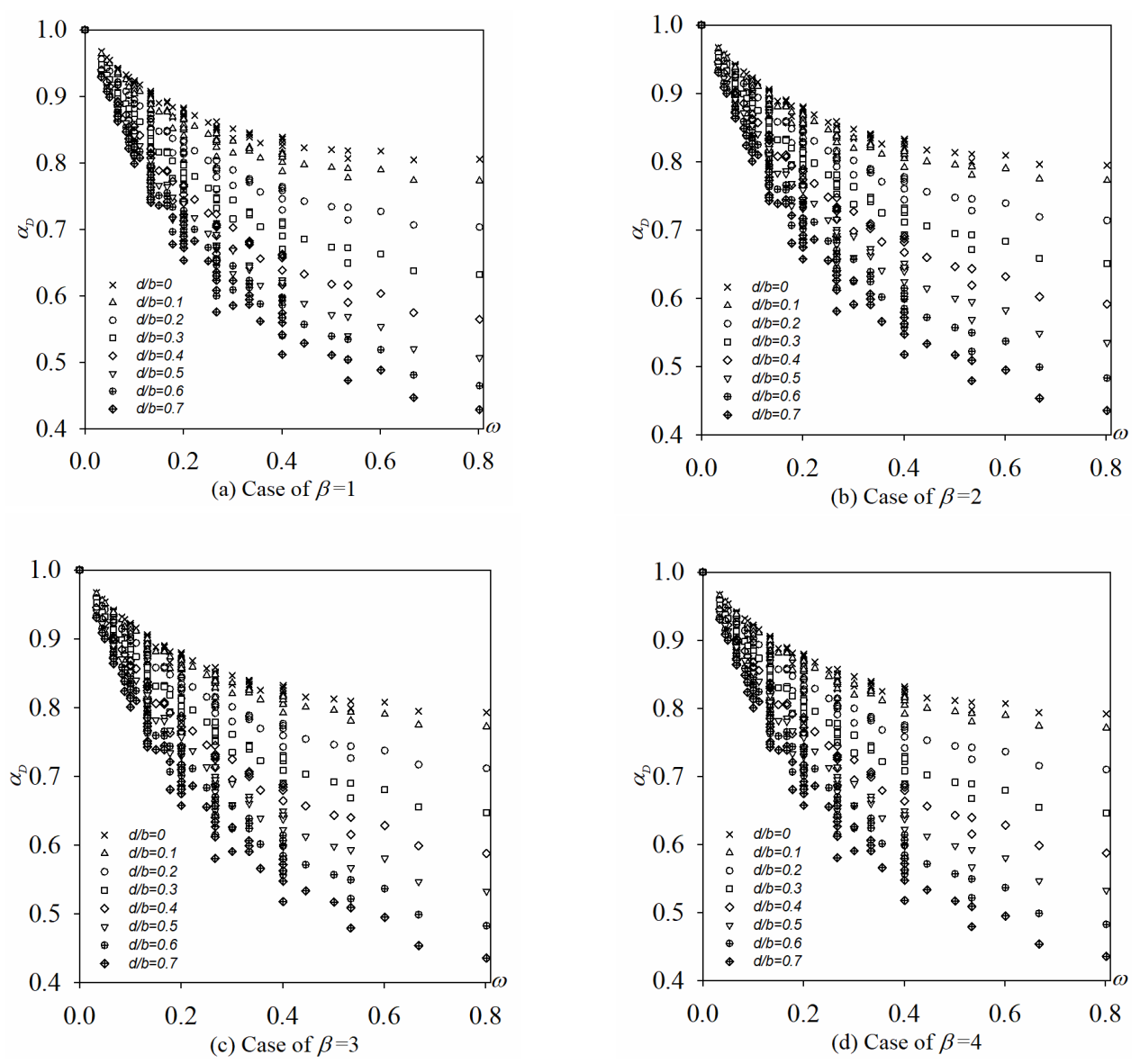

Figure 14. $\alpha_{D}$ vs. $\omega$ for perforated rectangular plate $(\beta=1,2,3,4)$ with the BCs of 4 S. 

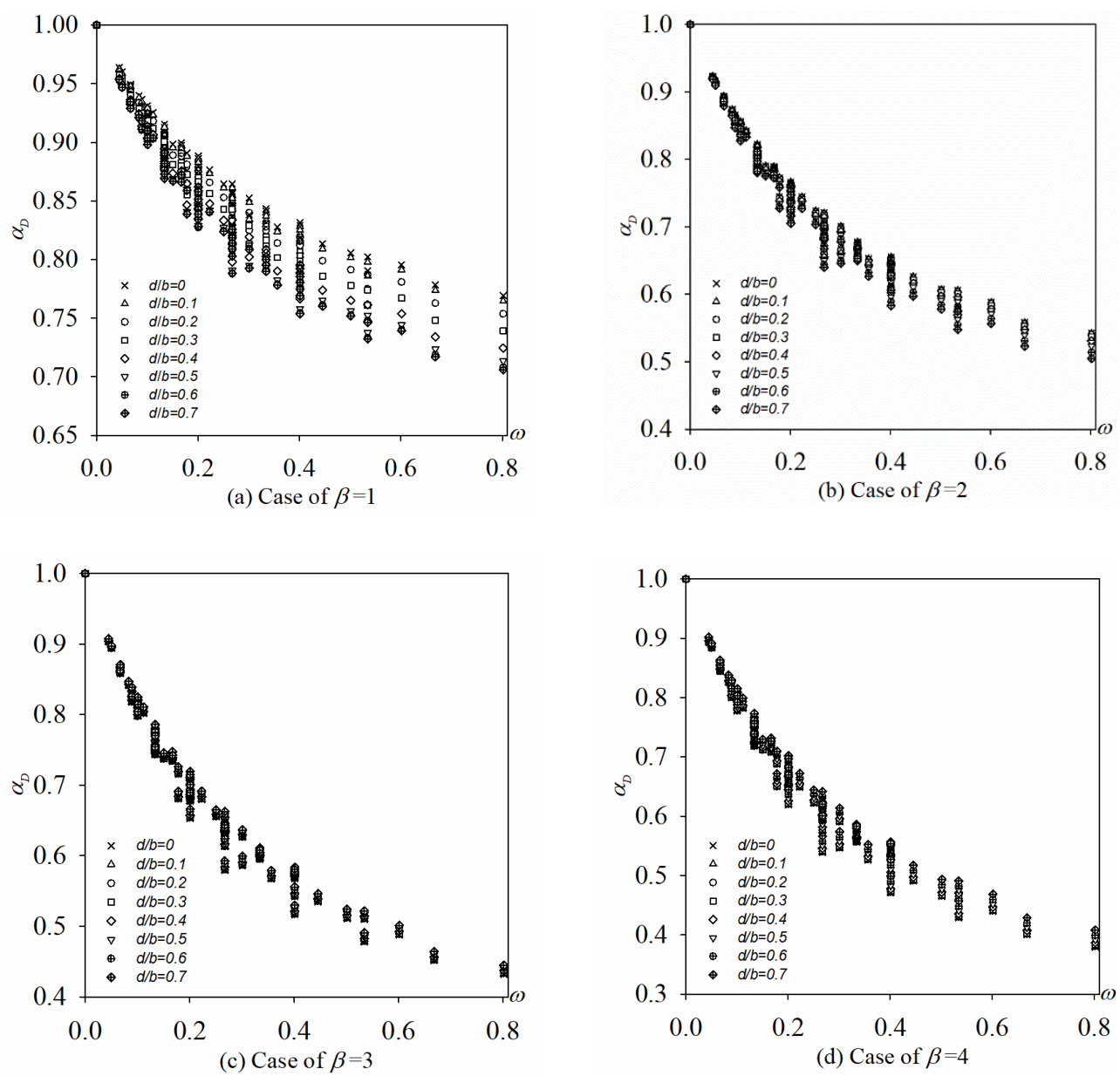

Figure 15. $\alpha_{D}$ vs. $\omega$ for perforated rectangular plate $(\beta=1,2,3,4)$ with the BCs of 3S1F.

\subsection{The Buckling Coefficient $K_{u}$}

\subsubsection{Hole Position Varying Along $x$-Axis}

Figure 16a d show the change in $K_{u}$ as hole position parameter $e_{x} / b$ is enlarged with the BCs of $4 \mathrm{~S}$, for different $d / b$ from 0 to 0.7 and different plate aspect ratios $\beta$ from 1 to 4 . It can be seen that hole position parameter $e_{x} / b$ has a little effect on $K_{u}$ for $d / b=0.1$, especially as the plate aspect ratios $\beta$ enlarged. It also can be seen that all the curves are very similar in trends and values with the same $d / b$ when $e_{x} / b$ is less than 0.5 . It means that when $\beta$ is an integer greater than 1 and $e_{x} / b$ is less than 0.5 , the buckling coefficient $K_{u}$ can be taken as a square plate with the same width $b$, the same hole $d / b$, and the same FRP pasting. It also can be seen from the figures that as $e_{x} / b$ is enlarged from 0 to $0.5, K_{u}$ decreases but gradual slow for $d / b$ is 0.1 , and first increases and then decrease for $d / b$ is 0.2 and 0.3 , and drastically increases for $d / b$ is from 0.4 to 0.7 . It means that when $d / b$ is bigger than 0.4 and $e_{x} / b$ is less than 0.5 , the buckling coefficient $K_{u}$ is very unstable, so it should be avoided to place big hole too close to the loading edge. For $e_{x} / b$ is bigger than $0.5, K_{u}$ shows little variation with $d / b$ and $e_{x} / b$, except when $\beta=2$ and $d / b$ is bigger than 0.4 . Figure $16 \mathrm{~b}$ shows that for $\beta=2$ and $e_{x} / b=1$, $K_{u}$ increases from 4.48 to 5.99 as $d / b$ is enlarged from 0.4 to 0.7 , this also proves the necessity of placing the big hole in the middle area of the plate.

Figure 17a d show the change in $K_{u}$ as hole position parameter $e_{x} / b$ is enlarged with the BCs of 3S1F , for different $d / b$ from 0 to 0.7 and different plate aspect ratios $\beta$ from 1 to 4 . In general, $K_{u}$ of plates with BCs of 3S1F is very small, and the curves shows that $K_{u}$ keeps almost constant irrespective of $e_{x} / b$ when $\beta=1,2$, and $\beta=3$ (or 4 ) with $d / b$ less than 0.3 . When $\beta=3$ (or 4 ) and 
$d / b \geq 0.3, K_{u}$ increases as the hole moves away from the loading edge towards its center along $x$-axis. Take $\beta=3$ and $d / b=0.7$ for example, as $e_{x} / b$ is enlarged from 0.375 to $1.5, K_{u}$ increases from 0.41 to 0.47 . Therefore, it is also recommended to place the big hole close to the middle area of the plate in $x$-axis with the BCs of 3S1F when $\beta$ is bigger than 3 .
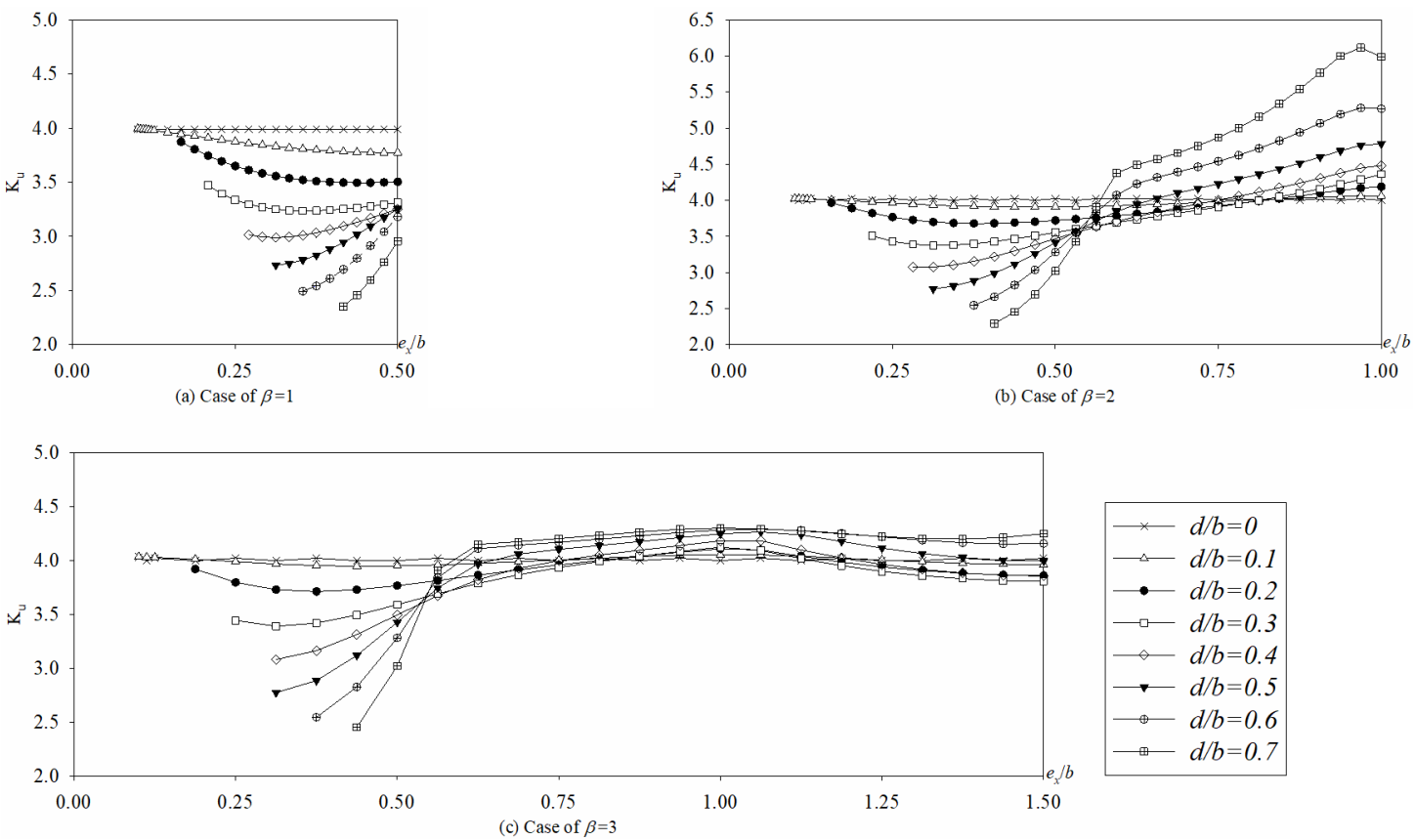

Figure 16. $K_{u}$ vs. $e_{x} / b$ for perforated rectangular plate $(\beta=1,2,3,4)$ with the BCs of 4 S.
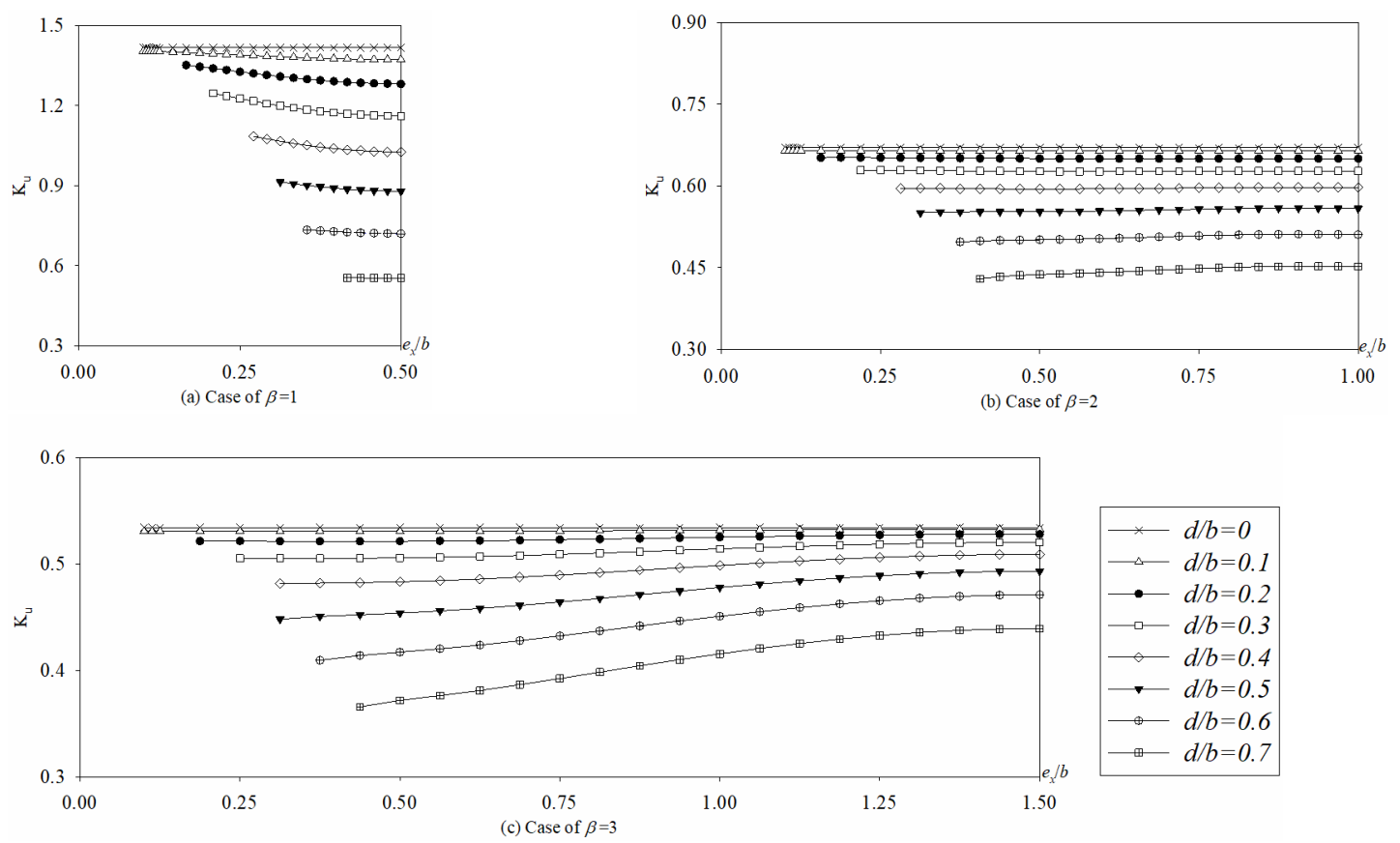

Figure 17. Cont. 


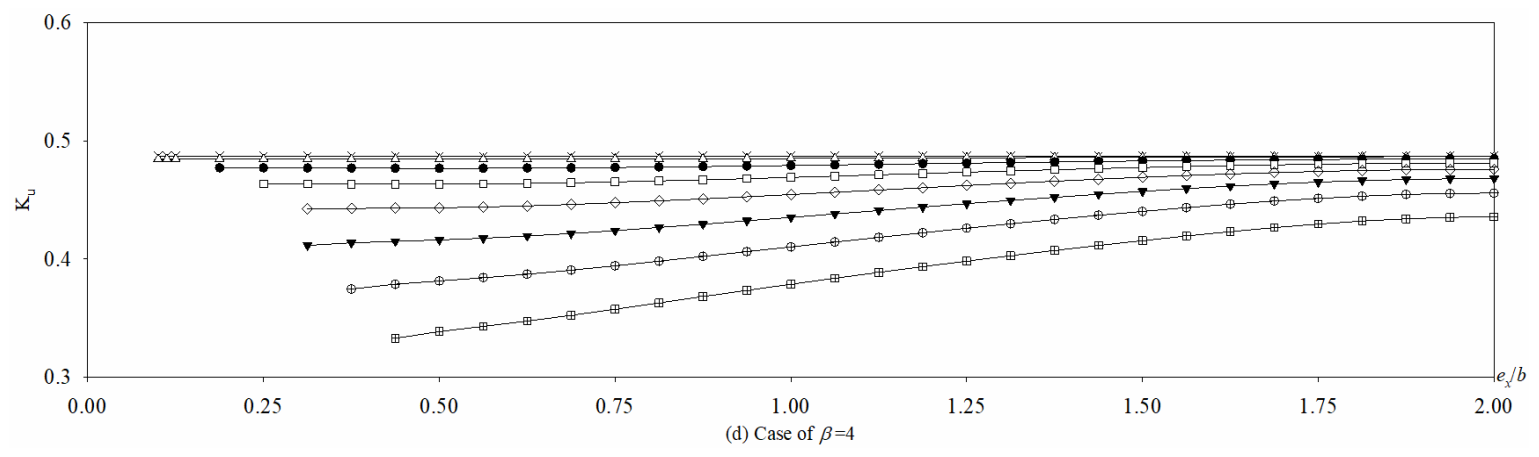

Figure 17. $K_{u}$ vs. $e_{x} / b$ for perforated rectangular plate $(\beta=1,2,3,4)$ with the BCs of 3S1F.

\subsubsection{Hole Position Varying Along $y$-Axis}

Figure 18 shows the change in $K_{u}$ as hole position parameter $e_{y} / b$ is enlarged from 0.5 to 0.9 with the $\mathrm{BCs}$ of $4 \mathrm{~S}$, for different $d / b$ from 0 to 0.7 , different plate aspect ratios $\beta$ from 1 to 4 , and different $e_{x} / b=0.5,1.0,1.5$, and 2.0. The center of the hole is halfway between nodal cross lines when $e_{x} / b=0.5$ and 1.5, and the center of the hole is at the nodal cross line when $e_{x} / b=1$ and 2. It could be noticed that for a small hole $d / b$ (e.g., $d / b=0.1$ and 0.2 ), there are little effect of the hole location $e_{y} / b$ on $K_{u}$, and the effect becomes more pronounced as hole size $d / b$ increases. When $d / b \geq 0.2$ in Figure $18 \mathrm{a} \sim \mathrm{d}$, it can be seen that the curves are very similar irrespective of $\beta$, that is: with the increasing of $e_{y} / b$, $K_{u}$ will decrease dramatically as $e_{y} / b$ is enlarged, especially when $d / b \geq 0.4$. Take $\beta=3, e_{x} / b=0.5$ and $d / b=0.6$ for example, as $e_{y} / b$ is enlarged from 0.5 to $0.65, K_{u}$ decreases from 3.28 to 2.20 , this decline is very severe. The curves with $e_{x} / b=1$ in Figure $18 \mathrm{e} \sim \mathrm{g}$ are also very similar, and the law of changes are similar to curves with $e_{x} / b=0.5$ (Figure 18a d). What's different is that for curves in Figure 18e $\sim$, when $e_{y} / b$ is less than $0.575, K_{u}$ is bigger than 4 which is the $K_{u}$ of plate without a hole. The law of changes in Figure $18 \mathrm{~h} \sim \mathrm{j}$ are between figures discussed above.

Figure 19 shows the change in $K_{u}$ as hole position parameter $e_{y} / b$ is enlarged from 0.05 to 0.95 with the BCs of 3S1F, for different $d / b$ from 0 to 0.7 and different plate aspect ratios $\beta$ from 1 to 4 . It can be seen that the $K_{u}$ of plates with $\mathrm{BCs}$ of $3 \mathrm{~S} 1 \mathrm{~F}$ is much less than those with BCs of $4 \mathrm{~S}$, especially when $\beta$ is bigger than 1 . The figure also shows that when the hole size $d / b$ is smaller than a specific value (for $\beta=1: 0.1 ; \beta=2: 0.1 ; \beta=3: 0.3 ; \beta=4: 0.4$ ), there is a little effect of the hole position $e_{y} / b$ on buckling coefficient $K_{u}$ and the $d / b$ effect becomes more pronounced as $d / b$ increases.

Over all, for a small hole size, there is little effect of the hole position $e_{y} / b$ on buckling coefficient $K_{u}$ regardless of the $\mathrm{BCs}(4 \mathrm{~S}$ or $3 \mathrm{~S} 1 \mathrm{~F})$, and becomes more pronounced as $d / b$ increases. The plate's stability improves as the hole places near the middle of the plate for $4 \mathrm{~S}$ and the simple support edge for 3S1F in $y$-axis. 


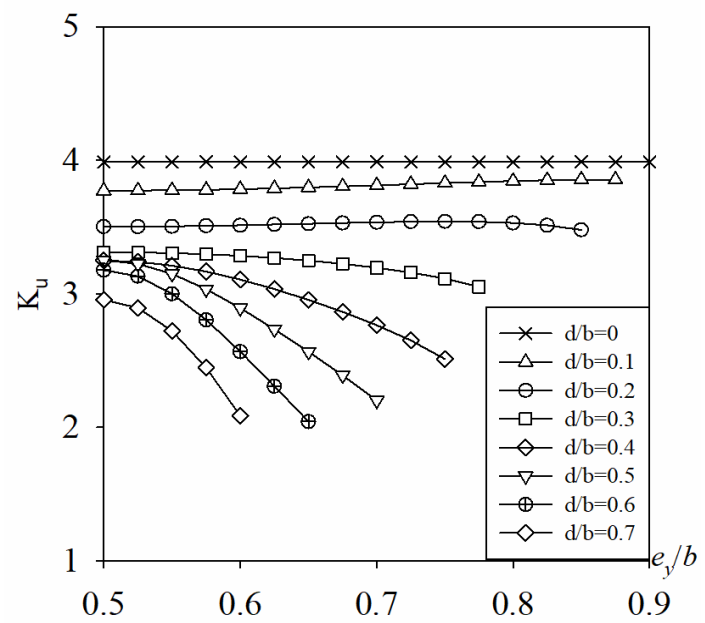

(a) Case of $\beta=1, e_{x} / \mathrm{b}=0.5$

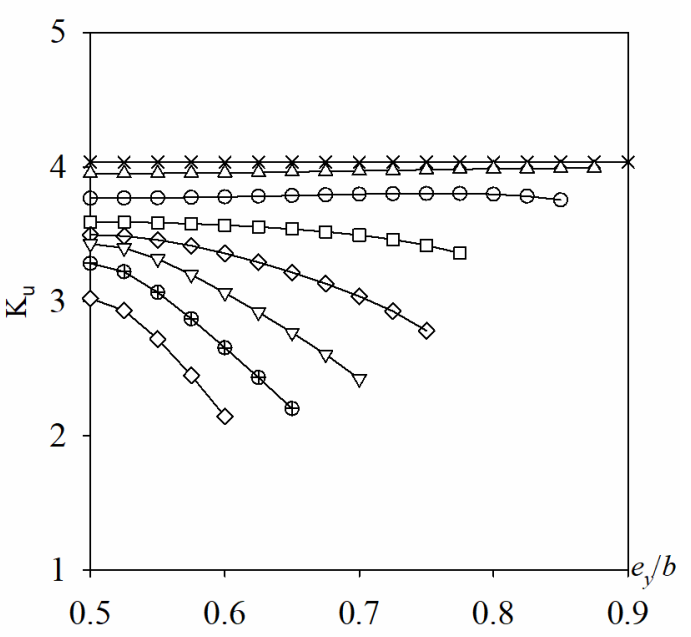

(c) Case of $\beta=3, e_{x} / \mathrm{b}=0.5$

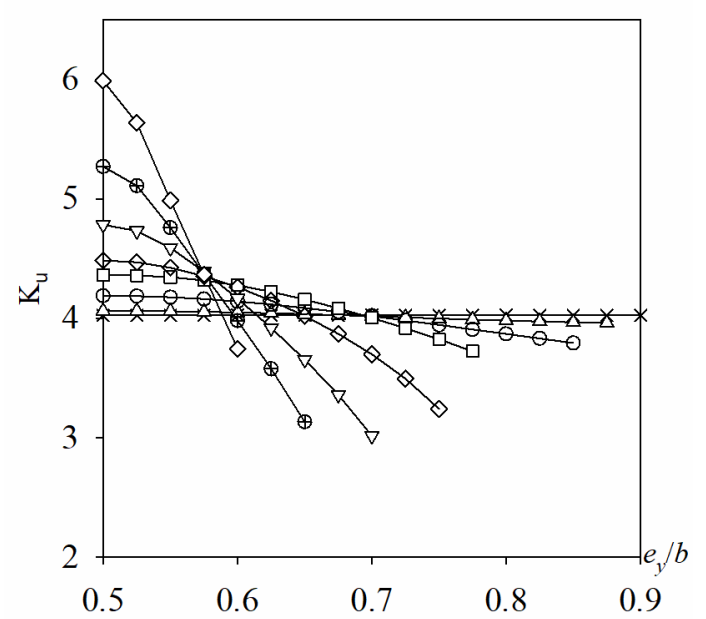

(e) Case of $\beta=2, e_{\gamma} / \mathrm{b}=1$

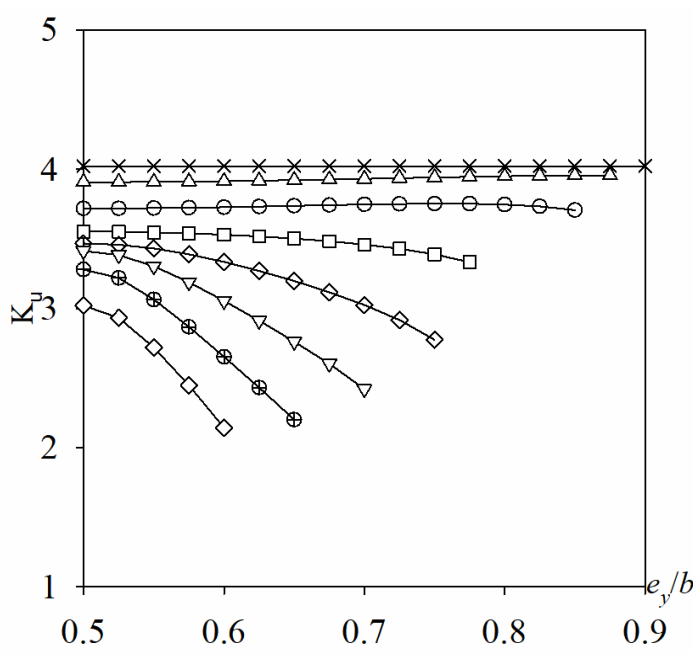

(b) Case of $\beta=2, e_{x} / \mathrm{b}=0.5$

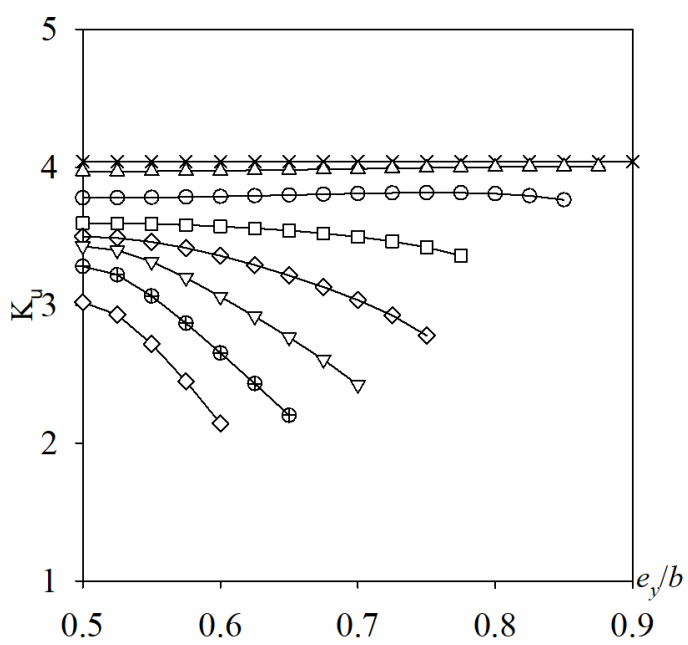

(d) Case of $\beta=4, e_{x} / b=0.5$

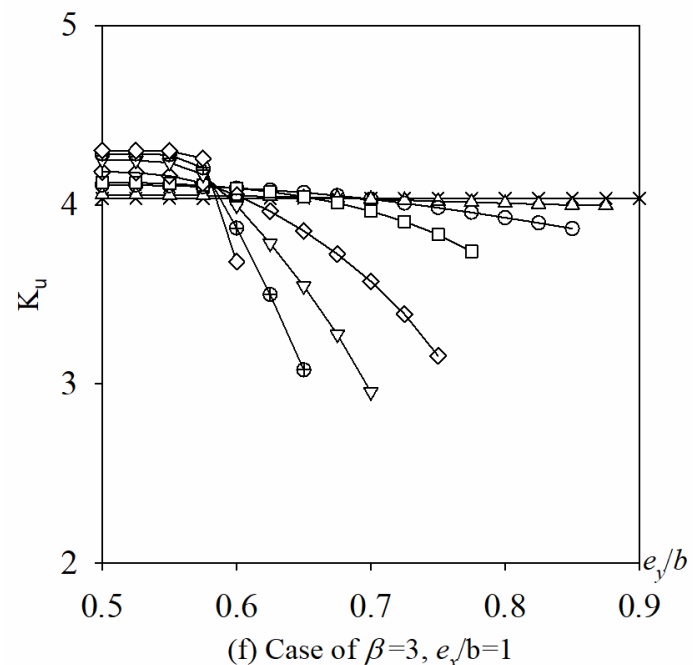

(f) Case of $\beta=3, e_{\gamma} / \mathrm{b}=1$

Figure 18. Cont. 


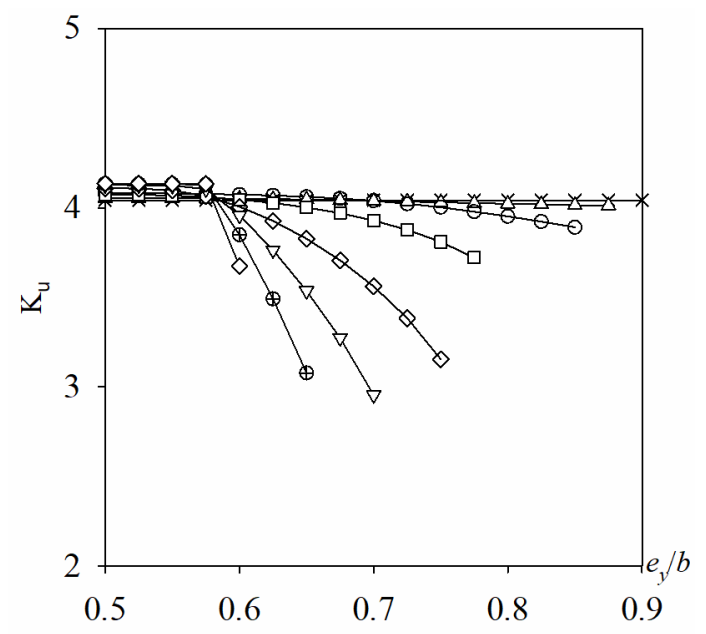

(g) Case of $\beta=4, e_{x} / \mathrm{b}=1$

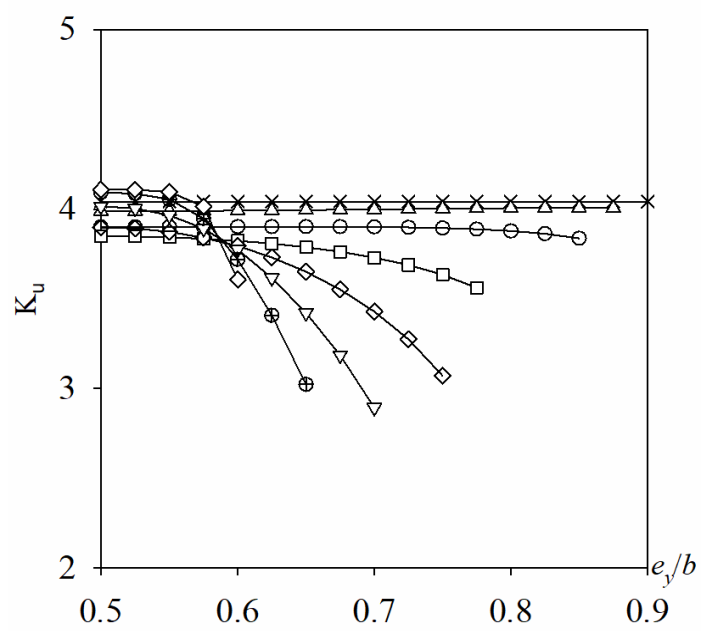

(i) Case of $\beta=4, e_{x^{\prime}} / \mathrm{b}=1.5$

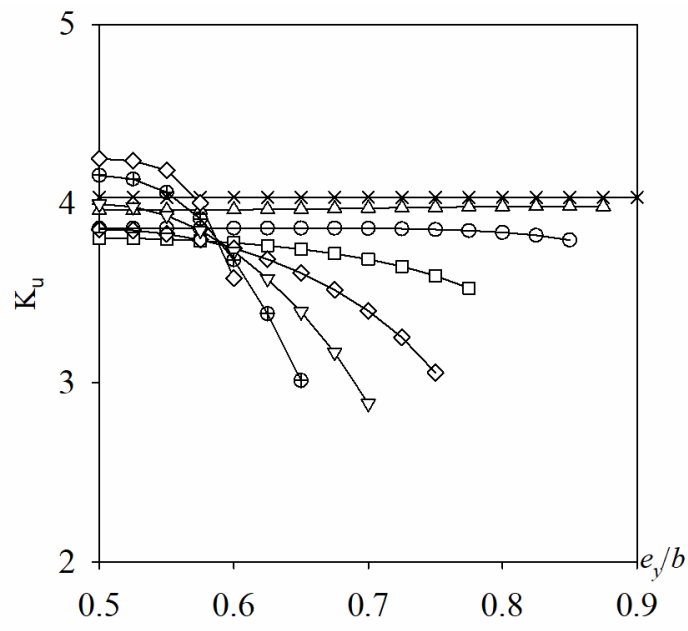

(h) Case of $\beta=3, e_{x} / b=1.5$

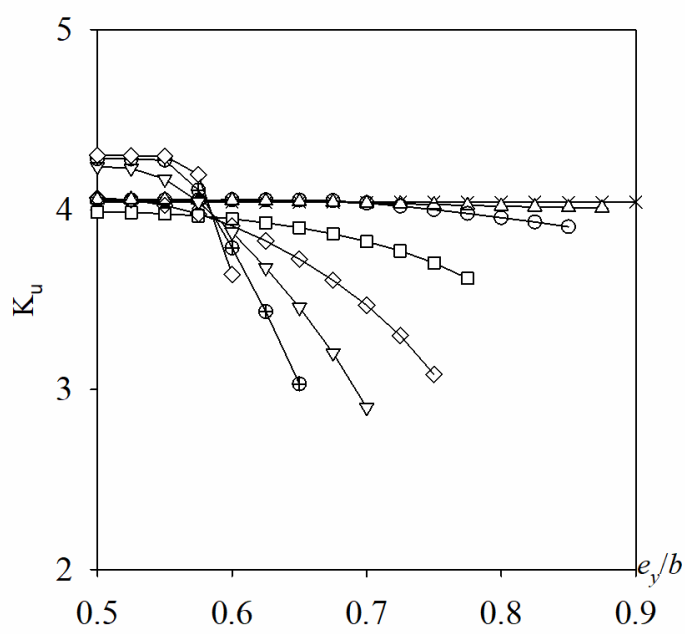

(j) Case of $\beta=4, e_{x} / \mathrm{b}=2$

Figure 18. $K_{u}$ vs. $e_{y} / b$ for perforated rect38angular plate $(\beta=1,2,3,4)$ with the BCs of 4 S.

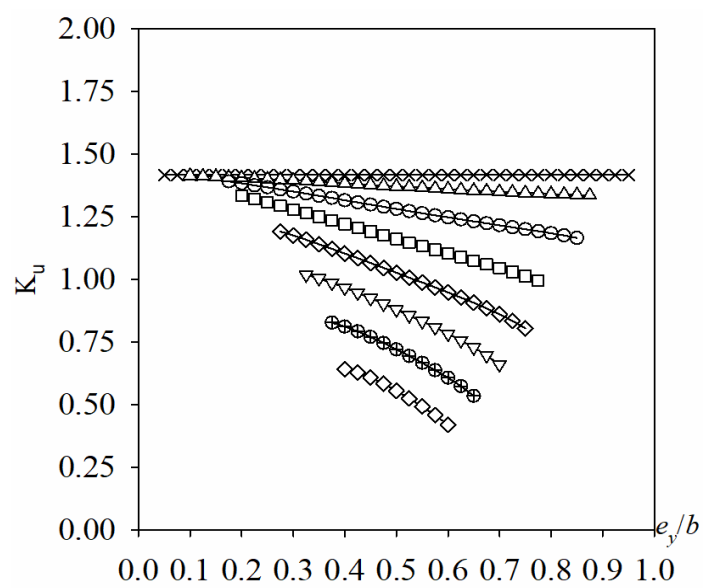

(a) Case of $\beta=1, e_{x} / \mathrm{b}=0.5$

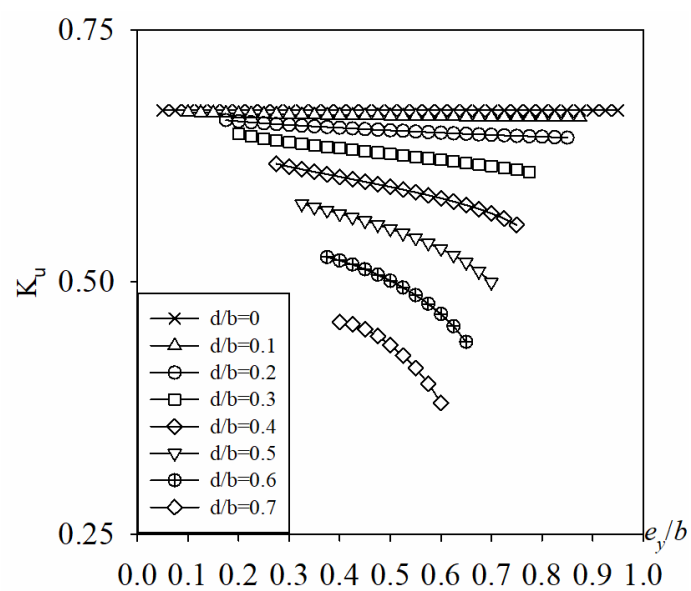

(b) Case of $\beta=2, e_{x} / \mathrm{b}=0.5$

Figure 19. Cont. 


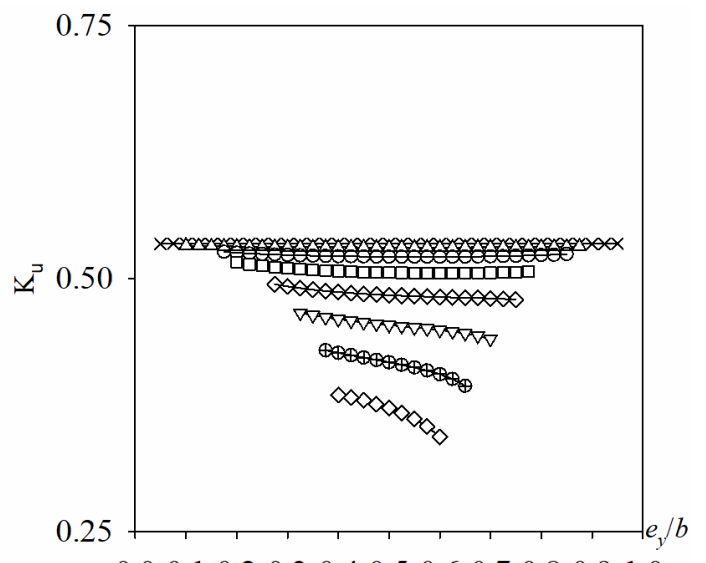

$\begin{array}{lllllllllllll}0.0 & 0.1 & 0.2 & 0.3 & 0.4 & 0.5 & 0.6 & 0.7 & 0.8 & 0.9 & 1.0\end{array}$

(c) Case of $\beta=3, e_{x} / \mathrm{b}=0.5$

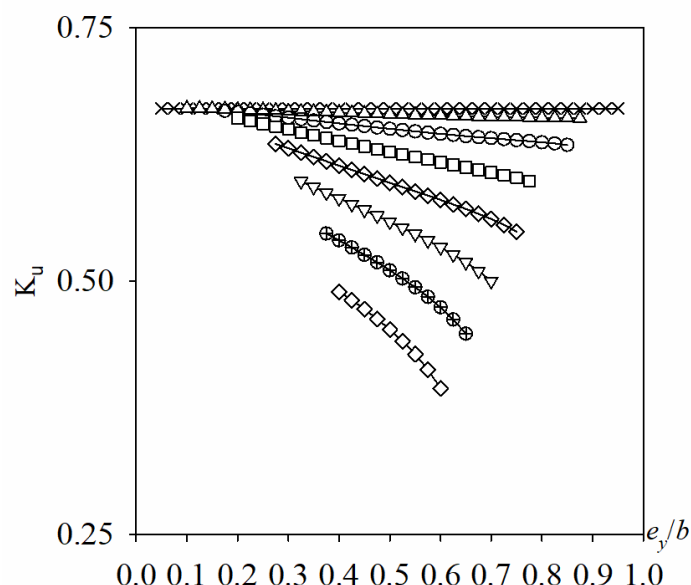

(e) Case of $\beta=2, e_{r} / \mathrm{b}=1$

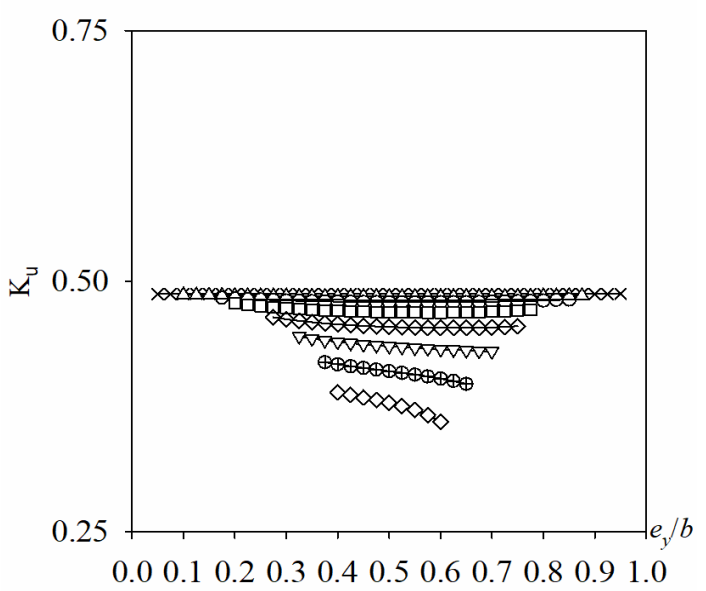

(g) Case of $\beta=4, e_{x} / \mathrm{b}=1$
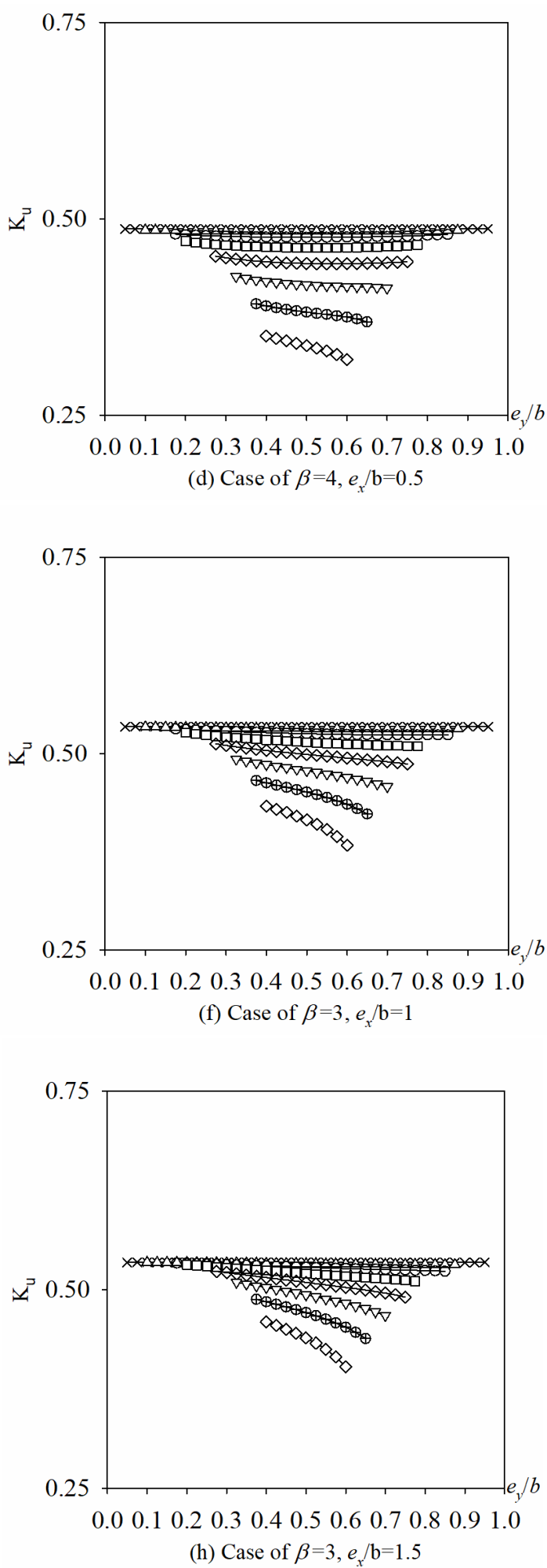

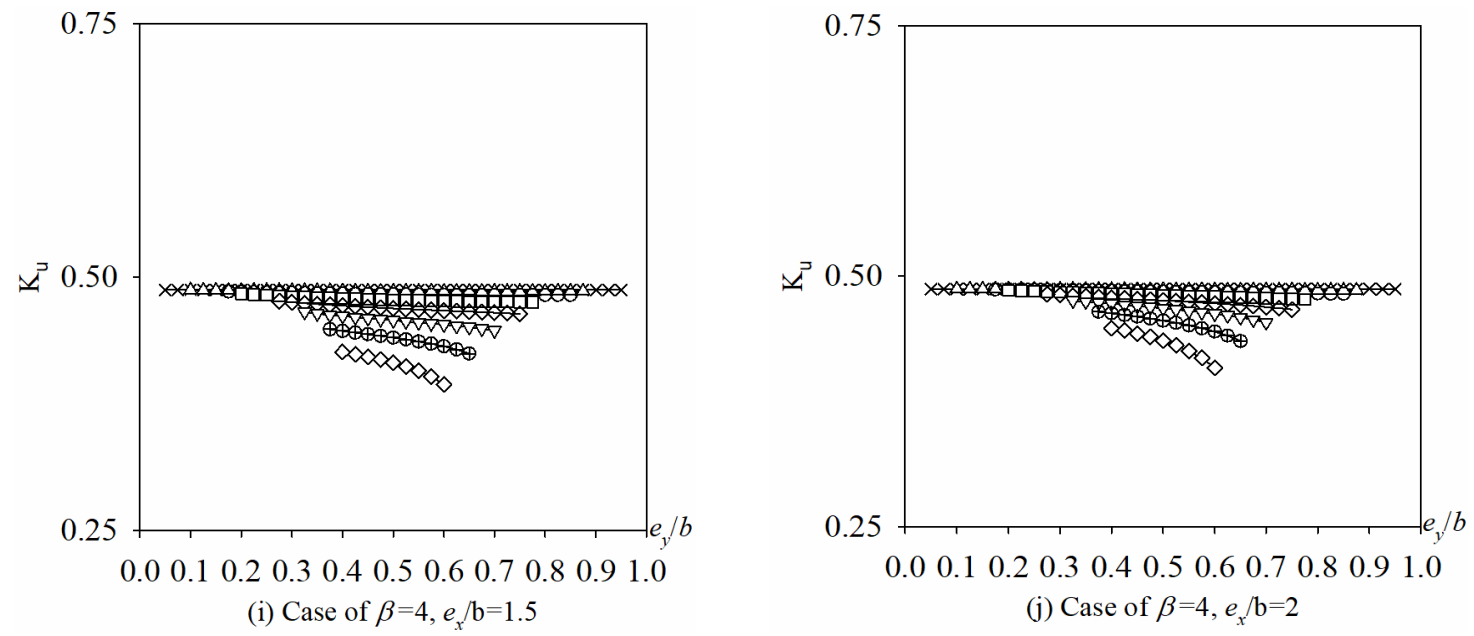

Figure 19. $K_{u}$ vs. $e_{y} / b$ for perforated rectangular plate $(\beta=1,2,3,4)$ with the BCs of 3S1F.

\subsubsection{Hole Size $d / b$}

Figure 20 show the change in $K_{u}$ as $d / b$ is enlarged from 0 to 0.7 with the BCs of $4 S$. When $e_{x} / b$ is 1 or 2 , the center of opening is in the node point. And when $e_{x} / b$ is 0.5 or 1.5 , the center of opening is in the middle of the node points. As shown Figure 20, two different behaviors can be seen depending on the hole in the node point or in the middle of the node points. When the hole is at the node point $\left(e_{x} / b\right.$ is 1 or 2), it is evident from the figure that $K_{u}$ show an increasing trend as $d / b$ is enlarged, for $\beta=2$, $K_{u}$ will increase from 4.02 to 5.99 as $d / b$ is enlarged from 0 to 0.7 , it is much better than that of all other cases. It also can be seen some discontinuities in the curves. Such discontinuities can be explained by the buckling mode shapes changing of the plate under compression, take $\beta=2$ and $e_{x} / b=1$ for example, when $d / b$ increases from 0 to 0.7 , the buckling mode has changed from 2 half-waves to 3 half-waves (seen from Figure 10). On the other hand, when the hole is in the middle of the node points ( $e_{x} / b$ is 0.5 or 1.5), $K_{u}$ of plates with $e_{x} / b=1.5$ first decrease slowly and then increase as $d / b$ is enlarged, and decreases gradually as $d / b$ is enlarged for plates with $e_{x} / b=0.5$. At the same time, we found that the $K_{u}$ of plates with $e_{x} / b=1.5$ are always bigger than those of plates with $e_{x} / b=0.5$. For a plate under uniaxial compression, the nodal cross-lines (the dotted line in the figures) act as imaginary supports for the plate in the lateral direction and the resistance to buckling is provided by the bending stiffness of the plate parts between these nodal cross lines, when the center of opening is in the node point, the bending stiffness will show little reduction, and when the hole is in the middle of the node points, it will reduce its bending stiffness and then reduce its buckling load and buckling coefficient, and $K_{u}$ will further reduce as $d / b$ increases, this can explain the phenomenon of $K_{u}$ with $e_{x} / b=1$ and 2 are bigger than those of plates with $e_{x} / b=0.5$ and 1.5. Therefore, it is recommend to put the hole in the node point in design.

Figure 21 show the change in $K_{u}$ as $d / b$ is enlarged from 0 to 0.7 with the BCs of 3S1F. It is obviously that the buckling coefficient $K_{u}$ of all the plates decrease as $d / b$ is enlarged, especially for $e_{x} / b=0.5$ and $\beta=1, K_{u}$ decreases from 1.42 to 0.55 as $d / b$ is enlarged from 0 to 0.7. Unlike the BCs of $4 \mathrm{~S}$, where the change rules of $K_{u}$ relies on $\beta$ and $e_{x} / b, K_{u}$ decreases rapidly as $d / b$ increases irrespective of $\beta$ and $e_{x} / b$ for plates with BCs of 3S1F, This phenomenon also can be explained as the buckling mode shapes are the same in all cases (seen from Figure 13). Meanwhile, by comparing Figure 21 with Figure 20, it is clear that $K_{u}$ of plates with BCs of 3S1F are far less than those of $4 \mathrm{~S}$. 


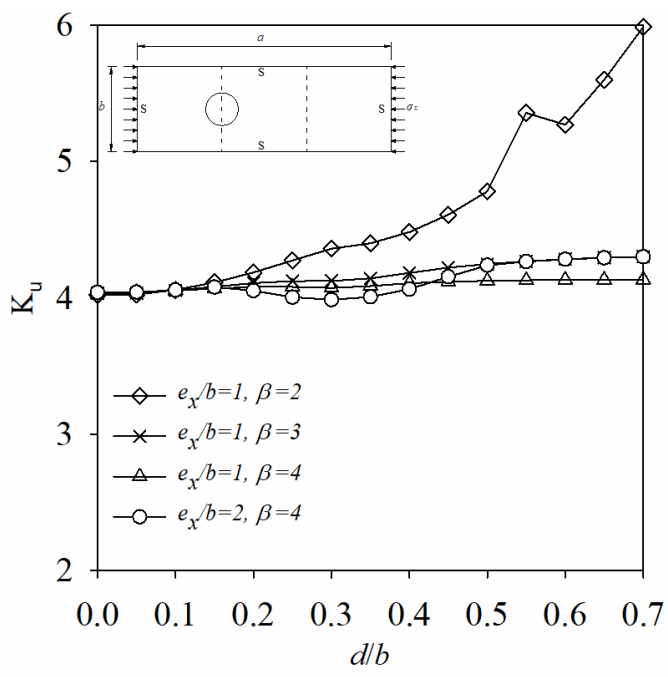

(a)

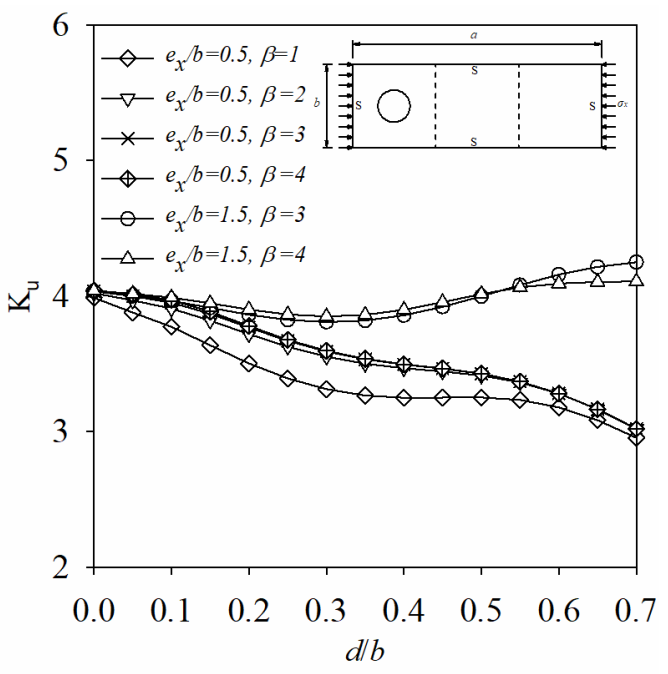

(b)

Figure 20. $K_{u}$ vs. $d / b$ with BCs of 4 S. (a): $e_{x} / b=1$ and $2 ;(\mathbf{b}): e_{x} / b=0.5$ and 1.5.

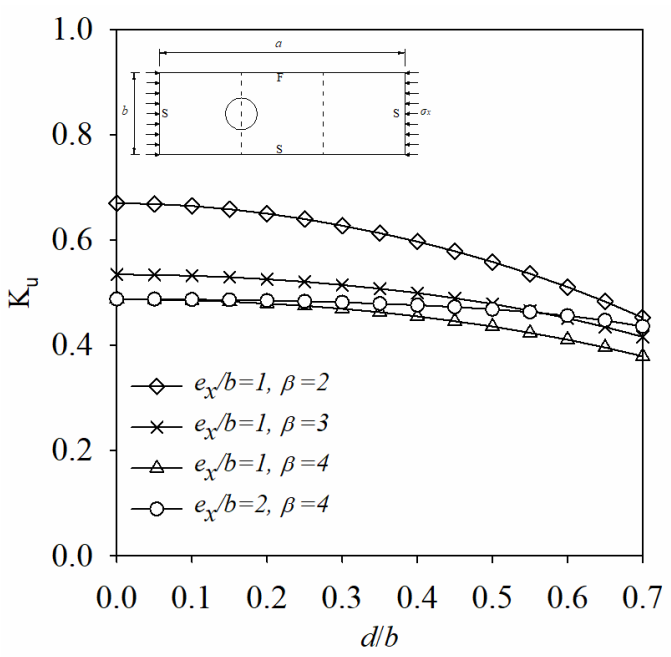

(a)

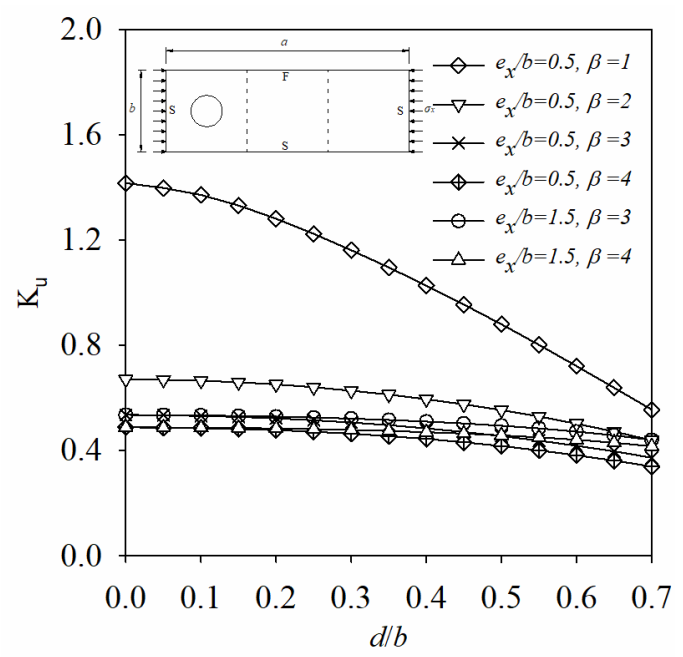

(b)

Figure 21. $K_{u}$ vs. $d / b$ with BCs of 3S1F. (a): $e_{x} / b=1$ and $2 ;(\mathbf{b}): e_{x} / b=0.5$ and 1.5.

\section{An Example for the Usage of the Proposed Method}

Example calculation for the ultimate capacity of a perforated plate strengthened with FRP under uniaxial compression, according to the winter equations in (22) (24), where $\rho$ is a slenderness reduction factor, $\lambda$ is the plate slenderness, $f_{y}$ is the yield stress of the steel, and $f_{c r}$ is the critical stress. $f_{c r}$ can be calculated using this method of this paper.

$$
\begin{gathered}
\rho=\frac{\lambda-0.22}{\lambda^{2}} \leq 1 \\
\lambda=\sqrt{\frac{f_{y}}{f_{c r}}} \\
P_{u}=\rho f_{y} b t_{t}
\end{gathered}
$$

The basic parameters of the example (Table 2, No. 7): 
$a=b=240 \mathrm{~mm}, d / b=0.1, e_{x} / b=0.5, e_{x} / b=0.5, t_{s}=2.61 \mathrm{~mm}, t_{f 0}=0.167 \mathrm{~mm}, n_{f}=1, E_{s}=2.0 \times 10^{5}$ $\mathrm{MPa}, E_{f}=2.6 \times 10^{5} \mathrm{MPa}, v_{s}=0.3, v_{f}=0.25, f_{y}=287.8 \mathrm{MPa}$, BCs: $4 \mathrm{~S}$.

The critical stress $f_{c r C a l}$ :

$$
\begin{aligned}
& t_{t}=2.61+0.167=2.777(\mathrm{~mm}), \alpha=\frac{E_{f} /\left(1-v_{f}^{2}\right)}{E_{s} /\left(1-v_{s}^{2}\right)}=1.225, \beta=\frac{t_{f}}{t_{s}}=0.064<0.2, \\
& D_{0}=C_{0}-B_{0}^{2} / A_{0}=E_{s} t_{s}^{3} / 12\left(1-v_{s}^{2}\right)=3.35 \mathrm{E} 5, D_{t} / D_{0}=1+3 \alpha \beta=1.24, D_{t}=4.14 \mathrm{E} 5, \\
& e_{x} / b=0.5, \beta=1, d / b=0.1 \text { from Figure } 20, K_{u}=3.77, \\
& \omega=\frac{n_{f} E_{f} t_{f 0}}{E_{s} t_{s}}=0.0784, \text { from Equation }(20), \alpha_{D}=(1.2 d / b+0.7) \omega^{2}-(1.6 d / b+0.74) \omega+ \\
& 1=0.947, \\
& f_{c r C a l}=\alpha_{D} K_{u} \frac{\pi^{2}}{t_{t} b^{2}} D_{t}=90.13(\mathrm{MPa}) .
\end{aligned}
$$

The ultimate capacity $\boldsymbol{P}_{u C a l}$ :

$$
\begin{aligned}
& f_{y}=287.8 \mathrm{MPa}, \lambda=\sqrt{\frac{f_{y}}{f_{c r}}}=1.79, \\
& \rho=\frac{\lambda-0.22}{\lambda^{2}}=0.491 \leq 1, \\
& P_{u C a l}=\rho f_{y} b t_{t}=94.12(\mathrm{KN}) .
\end{aligned}
$$

The experimental values of critical stress $f_{c r E x p}$ and ultimate load $P_{u \text { Exp }}$ are $92.87 \mathrm{MPa}$ and $96.64 \mathrm{KN}$, it is obvious that the values of $f_{c r C a l}$ and $P_{u C a l}$ are very close to $f_{c r E x p}$ and $P_{u E x p}$, respectively. Other plates are also calculated using this method and plotted in Table 2.

\section{Conclusions and Recommendations}

In order to study the elastic stability and propose a calculation method of perforated plates strengthened with FRP under uniaxial compression, series of FE models are built and several parameters are considered in the study: material's geometrical and mechanical properties, boundary conditions, plate aspect ratio, hole sizes, and hole position. A calculation method of buckling stress is proposed and modified based on the theory of composite plate and the numerical results. In addition to the detailed discussion and conclusions given in the previous section, some general conclusions and recommendations can be summarized as follows:

- The theory of composite plate proposed by Pister is available to calculate the flexural rigidity of the pure steel plate and the perfectly bonded layered plate, but it should be modified when calculating the flexural rigidity of the perfectly bonded layered plate due to not considered the orthotropic properties of FRP.

- The buckling coefficient $k$ needs to be modified as $K_{u}$ which considers the change of plate aspect ratio $\beta$, hole sizes $d / b$ and the hole position $e_{x}$ (or $e_{y}$ ).

- For BCs of $4 \mathrm{~S}$, when $e_{x} / b$ is an integer, the buckling mode shapes change as $d / b$ is enlarged, which cause some discontinuities in $K_{u}$ vs. $d / b$ curves (Figure 20). And for BCs of 3S1F, the buckling mode shapes is unchanged irrespective of $d / b$.

- For BCs of $4 S$, as $e_{x} / b$ is enlarged from 0 to $0.5, K_{u}$ decreases but gradual slow for $d / b$ is 0.1 , and first increases and then decrease for $d / b$ is 0.2 and 0.3 , and drastically increases for $d / b$ is enlarged from 0.4 to 0.7 . It means that when $d / b$ is bigger than 0.4 and $e_{x} / b$ is less than 0.5 , the buckling coefficient $K_{u}$ is very unstable, so it should be avoided to place big hole too close to the loading edge in $x$-axis, especially when $d / b$ is bigger than 0.4 .

- For BCs of 3S1F, $K_{u}$ is very small and stable irrespective of $e_{x} / b$ when $\beta=1,2$, and $\beta=3$ (or 4 ) with $d / b$ less than 0.4. When $\beta=3$ or 4 and $d / b$ is bigger than $0.4, K_{u}$ increases as the hole moves away from the loading edge towards its center along $x$-axis. Therefore, it is recommended to place the big hole $(d / b \geq 0.4)$ close to the middle area of the plate in $x$-axis, especially when $d / b$ is bigger than 0.4 . 
- For a small hole size, there is little effect of the hole position $e_{y} / b$ on buckling coefficient $K_{u}$ regardless of the $\mathrm{BCs}(4 \mathrm{~S}$ or $3 \mathrm{~S} 1 \mathrm{~F})$, and that effect becomes more pronounced as $d / b$ increases. So it is recommended to put the holes near the middle of the plate for $4 \mathrm{~S}$ and the simple support edge for 3S1F in $y$-axis.

- As shown in Table 2, the buckling stresses and ultimate loads calculated by the proposed method of this paper combining with the winter equation are very close to the experimental values.

Acknowledgments: The major source of funding for this project was provided by the School of Civil Engineering, Southeast University, China. The authors would also like to express their gratitude to National 973 Plan Program (No. 2012CB026200) for financial support and to Key Laboratory of Concrete and Prestressed Concrete Structures of Ministry of Education for technical support.

Author Contributions: Xin Tao, Long Zhang and Shuangyin Cao conceived and designed the experiments; Xin Tao and Long Zhang performed the experiments; Xin Tao, Shuangyin Cao and Long Zhang analyzed the data; Xin Tao and Long Zhang contributed analysis tools; Xin Tao wrote the paper. Xin Tao, Long Zhang and Shuangyin Cao have made substantial contributions to revise the manuscript.

Conflicts of Interest: The authors declare no conflict of interest.

\section{Glossary/Nomenclature/Abbreviations}

$a, b \quad$ the lengths in the longitudinal (transverse) directions

$t_{s} \quad$ the thicknesses of the steel plate

$t_{f} \quad$ the total thicknesses of FRP sheet

$n_{f} \quad$ FRP Layers' number

$t_{f 0} \quad$ single layer of FRP sheet's thickness

d diameter of the hole

$e_{x(y)} \quad$ the distance along $x(y)$-axis from the center of the hole to the left(bottom) edge

$E_{S}, E_{f} \quad$ the elastic modulus of steel and FRP

$v_{s}, v_{f} \quad$ the Poisson ratio of steel and FRP

$\beta \quad$ Plate aspect ratio, equal to $\mathrm{b} / \mathrm{a}$

$k \quad$ the buckling coefficient

$D_{0}, D_{t} \quad$ the flexural rigidity of plate without FRP and strengthened with FRP

$f_{c r} \quad$ the critical stress of the plate

$P_{c r} \quad$ the critical load of the plate

$P_{u} \quad$ the ultimate load of the plate

$\alpha \quad$ reinforcement index of FRP, calculated by Equation (8)

$\gamma \quad$ reinforcement index of FRP, calculated by Equation (9)

$\omega \quad$ reinforcement index of FRP, calculated by Equation (16)

$K_{u} \quad$ the modified buckling coefficient

\section{References}

1. El-Sawy, K.M.; Nazmy, A.S. Effect of aspect ratio on the elastic buckling of uniaxially loaded plates with eccentric holes. Thin Walled Struct. 2001, 39, 983-998. [CrossRef]

2. Shanmugam, N.E.; Thevendran, V.; Tan, Y.H. Design Formula for Axially Compressed Perforated Plates. Thin Walled Struct. 1999, 34, 1-20. [CrossRef]

3. Brown, C.J.; Yettram, A.L. The elastic stability of square perforated plates under combinations of bending, shear and direct load. Thin Walled Struct. 1986, 4, 239-246. [CrossRef]

4. Brown, C.J.; Yettram, A.L.; Burnett, M. Stability of plates with rectangular holes. J. Struct. Eng. 1987, 113, 1111-1116. [CrossRef]

5. Cheng, B.; Zhao, J. Strengthening of Perforated Plates under Uniaxial Compression: Buckling Analysis. Thin Walled Struct. 2010, 48, 905-914. [CrossRef]

6. Shaat, A.; Fam, A. Fiber-Element Model for Slender HSS Columns Retrofitted with Bonded High-Modulus Composites. J. Struct. Eng. 2007, 133, 85-95. [CrossRef]

7. Shaat, A.; Fam, A. Axial loading tests on short and long hollow structural steel columns retrofitted using carbon fibre reinforced polymers. Can. J. Civ. Eng. 2006, 33, 458-470. [CrossRef] 
8. Shaat, A.; Fam, A. Strengthening of short HSS steel columns using FRP sheets. In Proceedings of the 4th International Conference Advanced Composite Materials Bridges Structures, Calgary, AB, Canada, 20-23 July 2004.

9. Shaat, A.; Fam, A. Control of overall buckling of HSS slender steel columns using CFRP plates. In Proceedings of the First Asia Pacific conference FRP Structures (APFIS2007), Hong Kong, China, 12-14 December 2007; pp. 993-998.

10. Harries, K.A.; Peck, A.J.; Abraham, E.J. Enhancing stability of structural steel sections using FRP. Thin Walled Struct. 2009, 47, 1092-1101. [CrossRef]

11. Haedir, J.; Zhao, X.-L. Design of short CFRP-reinforced steel tubular columns. J. Construct. Steel Res. 2011, 67, 497-509. [CrossRef]

12. Bambach, M.R. Axial Capacity and Crushing of Thin-Walled Metal, Fibre-epoxy and Composite Metal-fibre Tubes. Thin Walled Struct. 2010, 48, 440-452. [CrossRef]

13. Bambach, M.R.; Elchalakani, M. Plastic Mechanism Analysis of Steel SHS Strengthened with CFRP under Large Axial Deformation. Thin Walled Struct. 2007, 45, 159-170. [CrossRef]

14. Bambach, M.R.; Jama, H.H.; Elchalakani, M. Axial Capacity and Design of Thin-Walled Steel SHS Strengthened with CFRP. Thin Walled Struct. 2009, 47, 1112-1121. [CrossRef]

15. Pister, K.S.; Dong, S.B. Elastic bending of layered plates. J. Eng. Mech. Div. 1959, 85, 1-10.

16. Timoshenko, S.P.; Gere, J.M. Theory of Elastic Stability; McGraw-Hill Book Company: New York, NY, USA, 1961.

17. Gerstle, K.H. Basic Structural Design; McGraw-Hill Book Company: New York, NY, USA, 1967.

18. Bambach, M. 6-Strengthening of Thin-Walled (Hollow) Steel Sections Using Fibre-Reinforced Polymer (FRP) Composites. In Rehabilitation of Metallic Civil Infrastructure Using Fiber Reinforced Polymer (FRP) Composites, 1st ed.; Karbhari, V.M., Ed.; Woodhead Publishing: Cambridge, UK, 2014; pp. 140-168. ISBN 9780857096654.

19. Tao, X.; Cao, S. Ultimate capacity of uniaxially compressed perforated steel plates strengthened by using CFRPs. In Civil, Architecture Environmental Engineering; CRC Press/Balkema: Leiden, The Netherlands, 2017; Volume 1, pp. 483-488. [CrossRef]

20. Narayanan, R.; Chow, F.Y. Ultimate Capacity of Uniaxially Compressed Perforated Plates. Thin Walled Struct. 1984, 2, 241-264. [CrossRef] 